\title{
The Determinants of Equity Risk and Their Forecasting Implications: A Quantile Regression Perspective
}

\author{
Giovanni Bonaccolto ${ }^{1,+}$ and Massimiliano Caporin ${ }^{2, *,+}$ \\ 1 Department of Economics and Management "Marco Fanno", University of Padova, Via del Santo 22, \\ 35123 Padova, Italy; giovanni.bonaccolto@unipd.it \\ 2 Department of Statistical Sciences, University of Padova, Via Cesare Battisti 241, 35121 Padova, Italy \\ * Correspondence: massimiliano.caporin@unipd.it; Tel.: +39-049-827-4199 \\ + These authors contributed equally to this work.
}

Academic Editor: Michael McAleer

Received: 21 May 2016; Accepted: 29 June 2016; Published: 7 July 2016

\begin{abstract}
Several market and macro-level variables influence the evolution of equity risk in addition to the well-known volatility persistence. However, the impact of those covariates might change depending on the risk level, being different between low and high volatility states. By combining equity risk estimates, obtained from the Realized Range Volatility, corrected for microstructure noise and jumps, and quantile regression methods, we evaluate the forecasting implications of the equity risk determinants in different volatility states and, without distributional assumptions on the realized range innovations, we recover both the points and the conditional distribution forecasts. In addition, we analyse how the the relationships among the involved variables evolve over time, through a rolling window procedure. The results show evidence of the selected variables' relevant impacts and, particularly during periods of market stress, highlight heterogeneous effects across quantiles.
\end{abstract}

Keywords: realized range volatility; quantile regression; volatility quantiles and density forecasting; forecast assessment

\section{Introduction}

Recent events, such as the subprime crisis, that originated in the United States and was marked by the Lehman Brothers' default in September 2008 and the sovereign debt crisis, that hit the Eurozone in 2009, have highlighted the fundamental importance of risk measurement, monitoring and forecasting. The volatility of asset returns, a commonly used measure of risk, is a key variable in several areas of finance and investment, such as risk management, asset allocation, pricing and trading strategies. Therefore, estimating and forecasting the volatility point values and distribution play a critical role. The use of predicted volatility levels is central, for instance, in the pricing of equity derivatives, in the development of equity derivative trading strategies and in risk measurement when risk is associated with volatility. The volatility distribution is of interest when trading/pricing volatility derivatives, when designing volatility hedges for generic portfolios and when accounting for the uncertainty of volatility point forecasts.

Many financial applications use constant volatility models [1], although empirical evidence suggests that variance changes over time. Several approaches have been developed with the purpose of achieving more accurate estimates, such as the class of autoregressive conditional heteroscedasticity (ARCH) [2] and generalized autoregressive heteroscedasticity (GARCH) [3] models and the stochastic volatility models [4-7]. Nevertheless, financial data are affected by several features, such as the so-called stylized facts [8], and standard GARCH and stochastic volatility models do not capture all of them [9]. 
We might also estimate volatility with non-parametric methods, such as by means of realized measures, which have been shown to perform better than traditional GARCH and stochastic volatility models when forecasting conditional second-order moments [10]. This approach has attracted considerable interest because of the availability of high-frequency financial data. In fact, as opposed to models that treat volatility as a latent (non-observable) element, realized measures use additional information from the intraday returns. We believe these methods provide more flexibility than standard GARCH-type models do, and therefore we focus on realized measures here. Among the various realized volatility measures, we choose a range-based measure for reasons of efficiency. Notably, the realized range estimator is five times more efficient than the corresponding realized variance [11]. This is because the realized range is built from two prices, the minimum and the maximum, for each intradaily interval, as opposed to using just the closing price in the realized volatility estimator. We therefore follow Christensen et al. [12] and adopt the realized range-based bias corrected bipower variation. Christensen et al. [12] demonstrated that their measure is a consistent estimator of the integrated variance in the presence of microstructure noise, arising from peculiar phenomena like non-continuous trading, infrequent trades and bid-ask bounce [13-15], and price jumps.

Studies that focus on volatility forecasting have identified key macroeconomic and financial variables as important drivers of volatility, highlighting their power in improving forecast performances. For instance, Christiansen et al. [16] predicted the asset return volatility using macroeconomic and financial variables in a Bayesian model averaging framework. They considered several asset classes, such as equities, foreign exchange, bonds and commodities, over long time spans and found that economic variables provide information about future volatility from both an in-sample and an out-of-sample perspective. Paye [17] tested the power of financial and economic variables to forecast the volatility at monthly and quarterly horizons and rarely found a statistical difference between the performance of macroeconomic fundamentals and univariate benchmarks. Fernandes et al. [18] used parametric and semi-parametric heterogeneous auto regressive (HAR) processes to model and forecast the CBOE Volatility Index (VIX) and found significant results using financial and macroeconomic variables as additional regressors. Caporin and Velo [19] used an HAR model with asymmetric effects with respect to volatility and return and GARCH and Glosten-Jagannathan-Runkle GARCH (GJR-GARCH) specifications for the variance equation. Caporin et al. [20] studied the relationship between the first principal component (FPC) of the volatility jumps, estimated using 36 stocks and a set of macroeconomic and financial variables, such as the VIX, the S\&P 500 volume, the Credit Default Swap (CDS) and the Federal Fund rates, and found that CDS captures a large part of the moves of the expected jumps. Opschoor et al. [21] used the Bloomberg Financial Conditions Index, which comprises money, bond and equity markets, observing that worse financial conditions are associated with both higher volatility and higher correlations. Given the findings in the previous studies, we take into account several macroeconomic and financial variables commonly used in the literature; nevertheless, among them, we select just the VIX and the S\&P 500 index, as all the other variables turned out to be non-significant in the model we propose.

Our purpose is to generalize the previous contributions further. We model and forecast the conditional quantiles of the realized range-based bias corrected bipower variation using the quantile regression method introduced by Koenker and Bassett [22]. Focusing on realized volatility measures, we believe that the quantile regression approach can provide useful new evidence by allowing the entire conditional distribution of the realized volatility measure to be estimated, instead of restricting attention to the conditional mean. This approach could have a relevant advantage when the impact of covariates is changing, depending on market conditions (say, on low or high volatility states) or when the purpose is to recover density forecasts without making a distributional assumption.

Other authors have applied quantile regression in a financial framework. For instance, Engle and Manganelli [23] proposed the Conditional Autoregressive Value at Risk (CAViaR) model to estimate the conditional value-at-risk, an important measure of risk that financial institutions and their regulators employ. In the present work, we do not consider the CAViaR model in order to 
control the computational complexity of the analysis. White et al. [24] generalized the CAViaR to the multi-quantile CAViaR (MQ-CAViaR) model, studying the conditional skewness and kurtosis of S\&P 500 daily returns. White et al. [25] extended the MQ-CAViaR model in the multivariate context to measure the systemic risk, a critical issue highlighted by the recent financial crises, taking into account the relationships among 230 financial institutions around the world. $\mathrm{Li}$ and Miu [26] proposed, on the basis of the binary quantile regression approach, a hybrid bankruptcy prediction model with dynamic loadings for both the accounting-ratio based and market-based information. Castro and Ferrari [27] used the $\triangle \mathrm{CoVaR}$ model as a tool for identifying/ranking systemically important institutions. Finally, Caporin et al. [28] adopted quantile regressions in detecting financial contagion across bond spreads in Europe.

Our work is closely related to the contribution of Zikes and Barunik [29]. However, our analysis differs in some important ways. First, Zikes and Barunik [29] estimated volatility using a realized measure that takes into account only the effects of jumps in the price process. In contrast, we use the realized range-based bias corrected bipower variation, which considers the impact of microstructure noise and that of jumps. To the best of our knowledge, quantile regression methods for the analysis of realized range volatility measures have never been used in the econometric and empirical financial literature, which provides a strong motivation for our study. Second, Zikes and Barunik [29] performed quantile forecasts, focusing on a few quantiles; we go farther by estimating a fine grid of quantiles in order to recover information about the entire realized volatility conditional distribution. Finally, on the basis of the obtained volatility quantiles, we build the entire conditional volatility density.

Our work takes an empiric point of view and focuses on the high-frequency data of 16 stocks issued by large-cap companies that operate in different economic sectors. All companies are quoted on the U.S. market. In the first step, we analyse the FPC of the estimated volatility as a summary of the 16 series (a kind of market factor).

Our first empirical contribution to the literature by showing that the VIX and the S\&P 500 index have a significant impact on volatility quantiles, that their impact changes across quantiles, becoming irrelevant in some cases, and that the significance and strength of the relationship changes over time. This last finding is particularly evident when we focus on turbulent market phases, as in these periods we note an increase in the impact of both the VIX and the S\&P 500 index. The last finding is particularly evident for high-volatility quantiles. The heterogeneity we observe across quantiles can also be interpreted as evidence against the location-shift hypothesis, that assumes homogeneous impacts of the covariates across the conditional quantiles of the response variable. Therefore, we highlight the benefits obtained through the quantile regression approach with respect to the classical least squares method, which focuses just on the conditional expected value of the volatility and is not able to capture the more interesting and significant relationships in its extreme conditional quantiles. This empirical evidence suggests that the uncertainty of volatility point forecasting, as measured by the influence of covariates on different volatility quantiles, changes depending on market states. Consequently, volatility forecast precision is ensured, as is the appropriateness of volatility density forecasting. Both of these elements have a central role in risk management and volatility hedging and trading, thus providing support for our analyses. In volatility hedging, or in the pricing of volatility derivatives, the most traditional measure is the realized volatility. However, as previously mentioned, the realized range estimator is more efficient and therefore provides more appropriate density forecasts as opposed to the realized variance density forecasts.

Our second empirical contribution comes from the single-asset analyses. We develop a specific model for each asset to determine how the features of the 16 companies affect the relationships among the variables involved. We find some heterogeneity in the assets' reactions to macro-level variables, such as the VIX and the S\&P 500 index, which holds across both time and volatility quantiles. However, we find overall confirmation of the findings associated with the FPC. Therefore, the relevance of the quantile-based covariate impact on volatility is of interest both at the aggregated and at the single-asset level. 
Our third empirical contribution stems from a forecasting exercise. We compare the quantile-based density forecasts to those of a benchmark model adapted to the realized range volatility mean and variance. The reference model combines an HAR structure of the realized volatility mean plus a GJR-GARCH [30] for the mean innovation variances. The HAR structure, inspired by the work of Corsi [9], captures the persistence of realized measures and is consistent with the presence of heterogeneous agents in the market, while the GJR-GARCH structure is coherent with the volatility of volatility hypothesis discussed in Corsi et al. [31]. We compare the benchmark model density forecasts and the quantile regression-based forecasts using the tests proposed by Berkowitz [32] and Amisano and Giacomini [33]. Moreover, by using a quantile-based loss function, we also consider the Diebold and Mariano [34] test. We stress that the Berkowitz [32] test allows for an absolute evaluation of the density forecasts provided by one model, while the Amisano and Giacomini [33] approach compares two competing models. The results confirm that our approach performs better, thus providing support for the use of quantile regression methods in all areas where volatility quantiles might have a role. Among the possible applications, we mention volatility trading and volatility hedging $[35,36]$, as well as the evaluation of uncertainty around volatility point forecasts. The latter could be of crucial importance if we plan to combine volatility point and density forecasts derived from realized volatility measures with returns-based volatility models, as in the high-frequency-based volatility (HEAVY) models [37].

The remainder of the paper is structured as follows. Section 2 includes the description of the data and of the conditioning variables. Section 3 presents the model we propose to forecast the range bipower variation conditional quantiles. Section 4 is devoted to the density forecast and predictive accuracy and provides the details about the tests we use. The results are analysed in Section 5, and Section 6 consists of concluding remarks.

\section{Realized Volatilities and Conditioning Variables}

The database we use includes stock prices recorded with a frequency of one minute, from 9:30 a.m. to 4:00 p.m. of every trading day between 2 January 2003 and 28 June 2013, inclusive. The equities analysed are those of large companies that operate in various economic sectors of the U.S. market: AT\&T Inc. (ATT), Bank of America (BAC), Boeing (BOI), Caterpillar, Inc. (CAT), Citigroup, Inc. (CTG), FedEx Corporation (FDX), Honeywell International, Inc. (HON), Hewlett-Packard Company (HPQ), International Business Machines Corp. (IBM), JPMorgan Chase \& Co. (JPM), Mondelez International, Inc. (MDZ), Pepsico, Inc. (PEP), The Procter \& Gamble Company (PRG), Time Warner, Inc. (TWX), Texas Instruments, Inc. (TXN) and Wells Fargo \& Company (WFC). The dataset is drawn from TickData. The prices are adjusted for extraordinary operations and filtered for errors, anomalies and outliers that arise from traders' activities (for additional details see the company website $h t t p: / / w w w . t i c k d a t a . c o m$ ).

From the high-frequency data described above and after computing the assets returns, we estimate the daily volatilities of the 16 assets using the realized range-based bias corrected bipower variation $\left(R R V_{B V B C}^{n, m}\right)$ introduced by Christensen et al. [12]. Notably, $R R V_{B V B C}^{n, m}$ is a robust estimator of the integrated variance in the the presence of both price jumps and microstructure noise. The realized range volatility sequences show that the financial companies are the most volatile, while all the companies show sudden increases in the risk measure during the global financial crisis. Detailed descriptive analysis and plots of the estimated realized range are available upon request.

A principal component analysis is carried out on the range-based bias corrected bipower variations of the 16 assets. In particular, the first principal component (FPC) explains $77 \%$ of the overall variance. The evolution of the assets' volatilities have a strong common behaviour that we might interpret as market or systematic behaviour. Therefore, the analysis of FPC could produce useful results. Consequently, in the following, we model the conditional quantiles of both FPC and the single-asset realized volatilities.

Previous studies in the literature, such as those by Caporin and Velo [19] and Caporin et al. [20], have used several indicators to analyse the realized variance and the range series. Therefore, in addition 
to the data described so far, we have taken into account some macroeconomic and financial variables, such as the VIX and the S\&P 500 indexes, the U.S. dollar-euro exchange rate, the crude oil price and the US Treasury interest rates, that convey important information about the overall market trend and risk. They will be considered exogenous variables that affect the conditional volatility quantiles. However, we included just two of them in our model: the daily return of the S\&P 500 index (sp500) and the logarithm of the VIX index (vix), as all the others turned out to be non-significant in the model we propose. $s p 500$ reflects the trend of the U.S. stock market, and we expect that negative returns of the S\&P 500 will have a positive impact on the market volatility, consistently to the well-known leverage effect. In contrast, high levels of vix reflect pessimism among the economic agents, so a positive relationship between vix and the volatility level is expected. The observations associated with sp500 and vix are recorded at a daily frequency and are recovered from Datastream.

Many macroeconomic and financial time series are not stationary and are often characterized by unit-root non-stationarity [38]. Building on this evidence, we must determine whether the data-generating process of vix is affected by unit-root (we do not report the tests on $s p 500$, as they provided no useful results: the index level is non-stationary, while the index return is stationary). To this purpose, we consider two standard tests: the augmented Dickey-Fuller (ADF) test [39] and the Phillips-Perron (PP) test [40]. The ADF test rejects the null hypothesis at the $10 \%$ significance level, and the test statistic equals -3.18 (with a $p$-value of 0.09). The PP test rejects the null hypothesis at the $5 \%$ level, and the test statistic is -24.48 (with a $p$-value of 0.03 ). Therefore, we have a moderate amount of evidence against the presence of a unit root for the vix series. The rejection of the null is much clearer with the PP test.

Beside vix and sp500, we use other quantities to describe the evolution of realized range conditional quantiles. First, we follow Corsi [9] in introducing among the explanatory variables those commonly adopted in (HAR) models of realized volatility. Focusing on the FPC of realized ranges, whose observed value at $t$ is denoted by $f p c_{t}$, a first explanatory variable is its lagged value, $f p c_{t-1}$. This variable is usually accompanied by other quantities that are built from local averages of past elements:

$$
\overline{f p c_{m^{*}} t}=\frac{1}{m^{*}} \sum_{i=0}^{m^{*}-1} f p c_{t-i}
$$

Corsi's [9] model used $m^{*}=5$ and $m^{*}=21$, representing the weekly and monthly horizons. These components allow the heterogeneous nature of the information arrivals [9] in the market to be considered. In fact, many operators have differing time horizons. For instance, intraday speculators have a short horizon, while insurance companies trade much less frequently. Therefore, agents whose time horizons differ perceive, react to and cause different types of volatility components. In our study, we use only $m^{*}=5$ with the first lag. In fact, the longer horizon component, with $m^{*}=21$, was not significant. We might interpret ${\overline{f p c_{5}-1}}_{t}$ as reflecting the medium-term investors who typically rebalance their positions at a weekly frequency. We found that $f p c_{t-1}$ and ${\overline{f p c_{5}}}_{t-1}$ are positively correlated with $f p c_{t}$, suggesting a positive impact at least on the mean. We will use similar variables (lagged and weekly elements) for each of the company-specific realized range sequences.

The last explanatory variable we consider, denoted by JUMP, takes into account the impact of jumps in the price process. At the single-asset level, the jump intensity could be detected through the test statistic introduced by Christensen and Podolskij [41], here denoted as $Z_{T P, t}$, by which we test the null hypothesis of no jumps at day $t$. For each asset, we test for the presence of jumps by computing the ratio between the number of days in which the null hypothesis of no jumps is rejected by the total number of days included in our dataset, with significance level set to 0.05 (the output of the test for jumps is available on request). We observed that the ratio ranges from $44.89 \%$ (CTG) to $91.97 \%$ (MDZ); therefore, the inferential procedure of Christensen and Podolskij [41] detects the presence of jumps, with clear heterogeneity across the 16 assets, and supports the inclusion of a component accounting for jumps in our model. In particular, we compute JUMP as the difference between the 
realized range-based variance [11,42], denoted as $R R V^{n, m}$, and the realized range-based bias corrected bipower variation [12], $R R V_{B V B C}^{n, m}$, which are, respectively, jump non-robust and jump robust estimators of the integrated variance. For the $i$-th stock, the observed value of JUMP at $t$ is denoted by jump $p_{i, t} ;$ we checked that the jump $p_{i, t}$ series has evident peaks in periods of financial turmoil (the estimated jumps series are available on request). For reasons of consistency, when we analyse $F P C$, we compute jump $p_{t}$ as the first principal component of jump $p_{i, t}$, for $i=1, \ldots, 16$.

To summarize, if we consider the FPC of the realized ranges, the variables of interest are: $f p c_{t}$ (i.e., the dependent variable), $f p c_{t-1},{\overline{f p c_{5}}}_{t-1}, v i x_{t-1}, s p 500_{t-1}$ and $j u m p_{t-1}$ (the explanatory variables). $f p c_{t-1}$ has the highest correlation with $f p c_{t}(0.75)$, and the signs of the correlation coefficients are consistent with the expectations, as $f p c_{t-1},{\overline{f p c_{5}}}_{t-1}$, vix $x_{t-1}$ and $j u m p_{t-1}$ are positively correlated with $f p c_{t}$, while $s p 500_{t-1}$ has a negative correlation coefficient. We obtain similar results for each realized volatility sequence where the lagged values, the weekly lags and the jump variable are company-specific (the results are not reported for space constraints but are available upon request).

\section{Modelling the Realized Range Conditional Quantiles}

We now introduce the model that we propose to study the conditional quantiles of volatility. As specified in the introduction, we focus on realized range quantiles, estimated using the quantile regression approach introduced by Koenker and Bassett [22]. Quantile regression overcomes some limitations of linear regression methods, including the sensitivity to outliers and the need to assume a linearity, and allows us to focus on the quantiles of the conditional distribution of a random variable without restricting the attention on the conditional mean. Let $Y$ be a real-valued random variable with distribution function $F_{Y}(y)=P(Y \leq y)$. For any $0<\tau<1$, the $\tau$-th quantile of $Y$ is equal to $F_{Y}^{-1}(\tau)=\inf \left\{y: F_{Y}(y) \geq \tau\right\}$. In our work, $Y$ coincides with FPC when we study the FPC of the stocks' volatilities; at the single-asset level it is equal to the volatility of each stock.

Let $I(\cdot)$ be the indicator function taking value 1 if the condition in $(\cdot)$ is true, 0 otherwise, and the approach introduced by Koenker and Bassett [22] makes use of the asymmetric loss function

$$
\rho_{\tau}(\epsilon)=\epsilon[\tau-I(\epsilon<0)],
$$

showing that the minimizer $\tilde{y}_{\tau}$ of the expected loss function $\mathbb{E}\left[\rho_{\tau}\left(Y-\tilde{y}_{\tau}\right)\right]$ satisfies $F_{Y}\left(\tilde{y}_{\tau}\right)-$ $\tau=0$. In particular, $\tilde{y}_{\tau}$ is the conditional quantile function $Q_{Y}\left(\tau \mid X_{1}, X_{2}, \ldots, X_{\delta}\right)$ in the linear quantile regression:

$$
\tilde{y}_{\tau}=Q_{Y}\left(\tau \mid X_{1}, X_{2}, \ldots, X_{\delta}\right)=\beta_{0}(\tau)+\beta_{1}(\tau) X_{1}+\ldots+\beta_{\delta}(\tau) X_{\delta}
$$

where $\mathbf{X}=\left(X_{1}, X_{2}, \ldots, X_{\delta}\right)$ is the vector of $\delta$ explanatory variables. When we analyse the FPC quantiles, the covariates are $f p c_{t-1},{\overline{f p c_{5-1}}}_{t}, v i x_{t-1}, s p 500_{t-1}$ and jump $p_{t-1}$; in contrast, at the single-asset level, the explanatory variables are $r r v_{i, t-1}, \overline{r r v} v_{i, t-1}, v i x_{t-1}, s p 500_{t-1}$ and jump $p_{i, t-1}$.

Given the time index $t=1, \ldots, T$, let $y_{t}$ and $x_{j, t}$ be, respectively, the realizations of $Y$ and $X_{j}$ for $j=1, \ldots, \delta$ at $t$. Then, the parameter vector $\boldsymbol{\beta}(\tau)=\left(\beta_{0}(\tau), \ldots, \beta_{\delta}(\tau)\right)$ is estimated as a solution of the quantile regression problem:

$$
\min _{\boldsymbol{\beta} \in \mathbb{R}^{\delta+1}} \sum_{t=1}^{T} \rho_{\tau}\left(y_{t}-\beta_{0}-\beta_{1} x_{1, t}-\ldots-\beta_{\delta} x_{\delta, t}\right) .
$$

The specification adopted for the conditional quantiles of the FPC is the following:

$$
\begin{aligned}
Q_{f p c_{t}}\left(\tau \mid \mathbf{x}_{t-1}\right) & =\mathbf{x}_{t-1}^{\prime} \boldsymbol{\beta}(\tau)=\beta_{0}(\tau)+\beta_{1}(\tau) f p c_{t-1}+\beta_{2}(\tau){\overline{f p c_{5}}}_{t-1}+\beta_{3}(\tau) v i x_{t-1} \\
& +\beta_{4}(\tau) s p 500_{t-1}+\beta_{5}(\tau) j u m p_{t-1}
\end{aligned}
$$


where $Q_{f p c_{t}}\left(\tau \mid \mathbf{x}_{t-1}\right)$ denotes the $\tau$-th quantile of $f p c_{t}$, conditional to the information included in $\mathbf{x}_{t-1}$. Although this approach is not novel, as conditional quantiles have already been used in a risk-management framework (e.g., Engle and Manganelli [23], White et al. [24] and White et al. [25]), we stress that, to the best of our knowledge, realized measures based on ranges have never been used. Therefore, even a simple estimation of Model (5) would provide useful results in terms of revealing the impact of covariates and the stability of the various coefficients across quantiles. Quantile regressions could also be used to forecast the conditional quantiles of the realized range volatility sequence, as we discuss below.

Certain events, such as the subprime crisis that originated in the U.S. and that was marked by the Lehman Brothers' default in September 2008 and the Eurozone sovereign debt crisis in 2010-2011, had considerable effects on the mechanisms that govern the international financial system. These extreme events could have affected the relationship between control variables and conditional quantiles, so it is necessary to determine whether the relationships that characterize Model (5) change over time before, during or after such periods of turmoil. For this purpose, we performed a rolling analysis with a step of one day and a window size of 500 observations. Thus, it is possible to determine how the coefficient values evolve over time and over $\tau$. Further, Engle and Manganelli [23], within a risk management perspective, adopt quantile regression methods for the estimation of the value-at-risk. In their model, they introduce lagged quantiles among the conditioning variables. We do not follow their approach to control the computational complexity given the presence of a rolling method in the evaluation of conditional quantiles.

We also built Model (5) to predict the conditional quantiles of the FPC computed on the realized range-based bias corrected bipower variations of the 16 assets. The forecasts, produced for a single step ahead, provide relevant details for the covariates' prediction abilities.

As the underlying companies have different features and operate in different economic sectors, it is also useful to build a model for each asset. These asset-specific models have the same structure of the one given in Equation (5), but the dependent variable is the conditional $R R V_{B V B C}^{n, m}$ quantile of the one asset, and $f p c_{t-1},{\overline{f p c_{5}}}_{t-1}$ and jump $p_{t-1}$ are replaced with the analogous quantities computed for each asset. Therefore, the model built for the $i$-th asset, for $i=1, \ldots, 16$, is

$$
\begin{aligned}
Q_{r r v}\left(\tau \mid \mathbf{x}_{i, t-1}\right) & =\beta_{0, i}(\tau)+\beta_{1, i}(\tau) r r v_{i, t-1}+\beta_{2, i}(\tau) \overline{r r v}_{5 i, t-1}+\beta_{3, i}(\tau) v i x_{t-1} \\
& +\beta_{4, i}(\tau){\operatorname{sp} 500_{t-1}+\beta_{5, i}(\tau) j u m p_{i, t-1}}
\end{aligned}
$$

where $r r v_{i, t}$ is the observed $R R V_{B V B C}^{n, m}$ related to the $i$-th company at day $t, \overline{r r v}_{5, t}$ is the mean of the $r r v_{i, t}$ values recorded in the last five days and jump $p_{i, t-1}$ is the difference between $R R V^{n, m}$ and $R R V_{B V B C}^{n, m}$ computed for the $i$-th stock at day $t-1$.

The models that we propose allow the conditional quantiles of a realized volatility measure to be estimated. We do not know the true, unobserved volatility of the assets' returns (they are estimated), so measurement errors might play a role. However, we restrict our attention to the forecast of the realized measure at a given sampling frequency and not to a forecast of the future returns' volatility. As a consequence, as Zikes and Barunik [29] discussed, the impact of measurement errors has limited importance.

\section{Density Forecast and Predictive Accuracy}

In contrast to Section 3, we prefer providing more details on the forecast evaluation tools that we consider given the central role of volatility forecasting in our work. In Section 3, we described the models that we propose to estimate the dynamic governing of the conditional quantiles of the response variable Y; that is, the FPC of the assets' volatilities in Model (5) or the volatilities of the single stocks in Model (6). Given the estimated model, we are able to forecast the conditional $\tau$-th quantile of $Y$, which is $Q_{y_{t}}\left(\tau \mid \mathbf{x}_{t-1}\right)$. 
The standard quantile regression approach allows estimating individual quantiles, but it does not guarantee their coherence, that is their increasing monotonicity in $\tau \in(0,1)$. For instance, it might occur that the predicted 95th percentile of the response variable is lower than the 90th percentile. If quantiles cross, corrections must be applied to obtain a valid conditional distribution of volatility. For instance, to cope with the crossing problem, Koenker [43] applied parallel quantile planes, whereas Bondell et al. [44] estimated the quantile regression coefficients with a constrained optimization method. Here, we follow a different approach proposed by Zhao [45]. Given a collection of $\vartheta$ predicted conditional quantiles $\left(Q_{y_{t}}\left(\tau_{1} \mid \mathbf{x}_{t-1}\right), \ldots, Q_{y_{t}}\left(\tau_{\vartheta} \mid \mathbf{x}_{t-1}\right)\right.$, for $0<\tau_{j}<\tau_{j+1}<1, j=1, \ldots, \vartheta-1$, we first rearrange them into ascending order, by making use of the quantile bootstrap method proposed by Chernozhukov et al. [46]. Then, starting from the rearranged quantiles, denoted by $\left(Q_{y_{t}}^{*}\left(\tau_{1} \mid \mathbf{x}_{t-1}\right), \ldots, Q_{y_{t}}^{*}\left(\tau_{\vartheta} \mid \mathbf{x}_{t-1}\right)\right)$, we estimate the entire conditional distribution with a nonparametric kernel method. The predicted density equals

$$
\hat{f}_{Y_{t}}\left(y^{*} \mid \mathbf{x}_{t-1}\right)=\frac{1}{\vartheta h_{\vartheta}} \sum_{i=1}^{\vartheta} K\left(\frac{y^{*}-Q_{y_{t}}^{*}\left(\tau_{i} \mid \mathbf{x}_{t-1}\right)}{h_{\vartheta}}\right),
$$

where $y^{*}$ are evenly interpolated points that generate the support of the estimated distribution, $h_{\vartheta}$ is the bandwidth, $K(\cdot)$ is the kernel function and $\hat{f}_{Y_{t}}\left(y^{*} \mid \mathbf{x}_{t-1}\right)$ is the one-period ahead forecasted density of $Y$ computed at $y^{*}$, given the information set available in $t-1$. Following Gaglianone and Lima [47], we use $K(\cdot)$ as the Epanechnikov kernel.

With the solution above described, we are able to recover the entire volatility density, which could be of interest if one is dealing with volatility trading or volatility hedging applications. In this case, the analysis takes a density-forecasting perspective, where the assessment of a proposed approach's predictive power (as compared to a benchmark model) and the evaluation of the potential benefits associated with introducing covariates are particularly important. Therefore, we apply three testing approaches: the tests proposed by Berkowitz [32] and Amisano and Giacomini [33] and a loss function-based forecast evaluation that builds on the Diebold and Mariano [34] testing approach.

If, for simplicity, we denote the ex ante forecasted conditional density $\hat{f}_{Y_{t}}\left(\cdot \mid \mathbf{x}_{t-1}\right)$ that we estimate in $t-1$ by $\hat{f}_{t-1}(\cdot)$, the first step of the Berkowitz [32] test consists in computing, for all the available days, the variable

$$
v_{t}=\int_{-\infty}^{y_{t}} \hat{f}_{t-1}(u) d u=\hat{F}_{t-1}\left(y_{t}\right)
$$

where $\hat{F}_{t-1}(\cdot)$ is the distribution function corresponding to the density $\hat{f}_{t-1}(\cdot)$.

Under correct model specification, Rosenblatt [48] showed that $v_{t}$ is i.i.d. and uniformly distributed on $(0,1)$, a result that holds regardless of the underlying distribution of $y_{t}$, even when $\hat{F}_{t-1}(\cdot)$ changes over time. Berkowitz [32] first pointed out that if $v_{t} \sim \mathcal{U}(0,1)$, then

$$
z_{t}=\Phi^{-1}\left(v_{t}\right) \sim \mathcal{N}(0,1)
$$

where $\Phi^{-1}(\cdot)$ denotes the inverse of the standard normal distribution function.

Given that under correct model specification $z_{t}$ should be independent and identically distributed as standard normal, an alternative hypothesis is that the mean and the variance differ from 0 and 1 , respectively, with a first-order autoregressive structure. In particular, Berkowitz [32] considered the model

$$
z_{t}-\mu_{b}=\rho_{b}\left(z_{t-1}-\mu_{b}\right)+e_{t}
$$

to test the null hypothesis $H_{0}: \mu_{b}=0, \rho_{b}=0, \operatorname{var}\left(e_{t}\right)=\sigma_{b}^{2}=1$. The test builds on (10) and is based on the likelihood-ratio statistic

$$
L R_{b}=-2\left[L_{b}\left(0,1,0 ; z_{t}\right)-L_{b}\left(\hat{\mu}_{b}, \hat{\sigma}_{b}, \hat{\rho}_{b} ; z_{t}\right)\right]
$$


where $L_{b}\left(\hat{\mu}_{b}, \hat{\sigma}_{b}, \hat{\rho}_{b} ; z_{t}\right)$ is the likelihood function associated with Equation (10) and is computed from the maximum-likelihood estimates of the unknown parameters $\mu_{b}, \sigma_{b}$ and $\rho_{b}$. Under the null hypothesis $H_{0}$, the test statistic is distributed as $\chi^{2}(3)$.

The Berkowitz [32] test can be applied to models that provide a density forecast for the realized range volatility. The alternative models' specifications for the conditional quantiles (such as with/without the covariates) or density forecast approaches may differ. Obviously, models that do not provide a rejection of the null hypothesis will be correctly specified, so, at least in principle, many alternative specifications could be appropriate for the data at hand.

The approach Berkowitz proposed allows for an absolute assessment of a given model. In fact, it focuses on the goodness of a specific sequence of density forecasts, relative to the unknown data-generating process. However, the Berkowitz test has a limitation in that it has power only with respect to misspecification of the first two moments. As Berkowitz [32] noted, if the first two conditional moments are specified correctly, then the likelihood function is maximized at the conditional moments' true values. Nevertheless, in practice, models could be misspecified even at higher-order moments. In that case, a viable solution is to compare density forecasts, that is, to perform a relative comparison given a specific measure of accuracy. To cope with this issue, in addition to the Berkowitz's [32] approach, we consider the Amisano and Giacomini's [33] test and a similar loss function-based approach that uses the Diebold and Mariano [34] test statistics.

Amisano and Giacomini [33] developed a formal out-of-sample test for ranking competing density forecasts that is valid under general conditions. The test is based on a widely adopted metric, the $\log$-score. In particular, the $\log$-score arising from our approach is equal to $\log \left(\hat{f}_{t-1}\left(y_{t}\right)\right)$, whereas $\log \left(\hat{g}_{t-1}\left(y_{t}\right)\right)$ is the $\log$-score obtained by a competing model. For the two sequences of density forecasts, we define the quantity

$$
W L R_{t}=w\left(y_{t}^{s t}\right)\left[\log \hat{f}_{t-1}\left(y_{t}\right)-\log \hat{g}_{t-1}\left(y_{t}\right)\right],
$$

where $y_{t}^{s t}$ is the realization of $Y$ at day $t$, standardized using the estimates of the unconditional mean and standard deviation computed from the same sample on which the density forecasts for $t$ are estimated, and $w\left(y_{t}^{s t}\right)$ is the weight the forecaster arbitrarily chooses to emphasize particular regions of the distribution's support. After computing the quantities $W L R_{t}$ for all of the samples considered in the forecast evaluation, we compute the mean $\overline{W L R}=(T-w s)^{-1} \sum_{t=w s+1}^{T} W L R_{t}$, where $w s$ is the window size adopted for the computation of density forecasts. The test can be used in the presence of a rolling approach for the computation of density forecasts. The value $w$ s indicates the size of the rolling window or the fact that time $t$ forecasts depend, at maximum, on the last $w s$ data points. To test for the null hypothesis of equal performance, that is, $H_{0}: E[\overline{W L R}]=0$, against the alternative of a different predictive ability $H_{1}: E[\overline{W L R}] \neq 0$, Amisano and Giacomini [33] proposed the use of a weighted likelihood ratio test:

$$
A G=\frac{\overline{W L R}}{\hat{\sigma}_{A G} / \sqrt{T-w s}}
$$

where $\hat{\sigma}_{A G}^{2}$ is a heteroskedasticity- and autocorrelation-consistent (HAC) Newey and West [49] estimator of the asymptotic variance $\sigma_{A G}^{2}=\operatorname{Var}[\sqrt{T-w s} \overline{W L R}]$. Amisano and Giacomini [33] showed that under the null hypothesis $A G \stackrel{d}{\rightarrow} \mathcal{N}(0,1)$.

We applied the Amisano and Giacomini [33] test by using four designs for the weights in Equation (12), which allows us to verify how the results change according to the particular regions of the distribution's support on which we are focusing. We set $w_{C E}\left(y_{t}^{s t}\right)=\phi\left(y_{t}^{s t}\right)$ to give a higher weight to the centre of the distribution, $w_{R T}\left(y_{t}^{s t}\right)=\Phi\left(y_{t}^{s t}\right)$ when we focus more on the right tail, $w_{L T}\left(y_{t}^{s t}\right)=1-\Phi\left(y_{t}^{s t}\right)$ for the left tail and $w_{N W}\left(y_{t}^{s t}\right)=1$ when giving equal importance to the entire support $(\phi($.$) and \Phi($.$) denote the standard normal density function and the standard normal$ distribution function, respectively). 
Finally, we carried out a comparison at the single quantile level, focusing on the quantiles that have critical importance in our framework. Therefore, we built on the approach Diebold and Mariano [34] proposed and considered the following loss function:

$$
\begin{aligned}
L_{\tau, t}^{(i)}\left(y_{t}, Q_{y_{t}}^{(i)}\left(\tau, \mathbf{x}_{t-1}\right)\right) & =\left[\tau-I\left(y_{t}-Q_{y_{t}}^{(i)}\left(\tau, \mathbf{x}_{t-1}\right)<0\right)\right] \\
& \times\left(y_{t}-Q_{y_{t}}^{(i)}\left(\tau, \mathbf{x}_{t-1}\right)\right),
\end{aligned}
$$

where $Q_{y_{t}}^{(i)}\left(\tau, \mathbf{x}_{t-1}\right)$ is the $\tau$-th forecasted quantile of $Y$, obtained from the $i$-th model.

Let $d_{D M, \tau, t}$ be the loss differential between the quantile forecasts from two competitive models, $i$ and $j$ (where $i$ represents our proposal), that is, $d_{D M, \tau, t}=L_{\tau, t}^{(i)}\left(y_{t}, Q_{y_{t}}^{(i)}\left(\tau, \mathbf{x}_{t-1}\right)\right)-$ $L_{\tau, t}^{(j)}\left(y_{t}, Q_{y_{t}}^{(j)}\left(\tau, \mathbf{x}_{t-1}\right)\right)$. After computing the quantities $d_{D M, \tau, t}$ for the forecasting sample, we compute the mean: $\overline{d_{D M, \tau}}=(T-w s)^{-1} \sum_{t=w s+1}^{T} d_{D M, \tau, t}$. We are interested in testing the null hypothesis $H_{0}: E\left[\overline{d_{D M, \tau}}\right]=0$ against the alternative $H_{1}: E\left[\overline{d_{D M, \tau}}\right] \neq 0$. Therefore, we compute for $\tau=\{0.1,0.5,0.9\}$ the test statistic Diebold and Mariano [34] proposed:

$$
D M_{\tau}=\frac{\overline{d_{D M, \tau}}}{\hat{\sigma}_{D M} / \sqrt{T-w s}}
$$

where $\hat{\sigma}_{D M}^{2}$ is a consistent estimate of $\sigma_{D M}^{2}=\operatorname{Var}\left(\sqrt{T-w s} \overline{d_{D M, \tau}}\right)$, the asymptotic (long-run) variance. Diebold and Mariano [34] showed that, under the null hypothesis of equal predictive accuracy, $D M_{\tau} \stackrel{d}{\rightarrow} \mathcal{N}(0,1)$.

\section{Empirical Results}

\subsection{Full-Sample Analyses}

First, we focus on Model (5) to analyse the full-sample estimated parameters and their $p$-values. This model was built to analyse and forecast the conditional quantiles of the FPC of the realized range-based bias corrected bipower variations. For simplicity, Table 1 shows just the results associated with $\tau=\{0.1,0.5,0.9\}$; additional results for other quantiles are available on request. The standard errors are computed by means of a bootstrapping procedure using the $x y$-pair method, which provides accurate results without assuming any particular distribution for the error term.

When $\tau$ equals 0.1 , only $s p 500_{t-1}$ is not significant at the $5 \%$ level. At $\tau=\{0.2,0.3,0.4,0.5\}$, all the coefficients have small $p$-values, while for $\tau=\{0.6,0.7\}$ only jump $p_{t-1}$ is not significant. Finally, when $\tau>0.7$, only $f p c_{t-1}$ and $s p 500_{t-1}$ are highly significant. Therefore, the first important result is that the variables that significantly affect $Q_{f p c_{t}}\left(\tau \mid \mathbf{x}_{t-1}\right)$ change according to the $\tau$ level. Notably, only $f p c_{t-1}$ is always significant, whereas $s p 500_{t-1}$ is not significant only at $\tau=0.1$. It is important to highlight that only $f p c_{t-1}$ and $s p 500_{t-1}$ are significant in order to explain the high quantiles of volatility, which assume critical importance in finance. Moreover, the fact that jump $p_{t-1}$ is not significant for high values of $\tau$ is a reasonable result because the volatility is already in a 'high' state, and we might safely assume that the jump risk is already incorporated in it.

The fact that $f p c_{t-1}$ and $s p 500_{t-1}$ are the most significant variables along the different quantiles' levels is further confirmed when we compare two different models: a restricted model that has fewer explanatory variables (just $f p c_{t-1}$ and $s p 500_{t-1}$ ) against an unrestricted one that includes all the available covariates. The comparisons are made by means of the pseudo-coefficient of determination proposed by Koenker and Machado [50], here denoted by $R^{1}(\tau)$, and the test statistic $\xi_{w}$ proposed by Koenker and Bassett [51]. $R^{1}(\tau)$ is a local goodness-of-fit measure, which ranges between 0 (when the covariates are useless to predict the response quantiles) and 1 (in the case of a perfect fit); with 
$\xi_{w}$, we aim to test the null hypothesis that the additional variables used in the unrestricted model do not significantly improve the goodness-of-fit with respect to the restricted model. Table 2 shows the values of the pseudo-coefficient of determination computed for the restricted and the unrestricted models at $\tau=\{0.1,0.2,0.3,0.4,0.5,0.6,0.7,0.8,0.9\}$. We first observe that $R^{1}(\tau)$ is a positive function of $\tau$ for both the models and then note that the differences between the restricted and the unrestricted models decrease as $\tau$ increases, to substantially disappear at $\tau=0.9$. Therefore, the contribution of $\overline{f p c}_{t-1}$, vix $x_{t-1}$ and jump $p_{t-1}$ to the goodness-of-fit of Model (5) is largely irrelevant at $\tau=0.9$. We obtain similar conclusions from the test proposed in Koenker and Bassett [51]: the null hypothesis of the test is not rejected at $\tau=0.9$, while at lower quantiles some of the additional variables provide sensible improvements in the model fit (see the fourth column of Table 2).

Table 1. Quantile regression results.

\begin{tabular}{|c|c|c|}
\hline Variable & Coefficient Value & $p$-Value \\
\hline \multicolumn{3}{|c|}{$\tau=0.1$} \\
\hline$f p c_{t-1}$ & 0.24157 & 0.00548 \\
\hline${\overline{f p c_{5}-1}}$ & 0.15385 & 0.03927 \\
\hline$v i x_{t-1}$ & 0.00020 & 0.00001 \\
\hline$s p 500_{t-1}$ & -0.00119 & 0.17431 \\
\hline$j u m p_{t-1}$ & 0.87638 & 0.03242 \\
\hline \multicolumn{3}{|c|}{$\tau=0.5$} \\
\hline$f p c_{t-1}$ & 0.44580 & 0.00000 \\
\hline${\overline{f p c_{5}-1}}$ & 0.28779 & 0.00013 \\
\hline$v_{i x-1}$ & 0.00019 & 0.00006 \\
\hline$s p 500_{t-1}$ & -0.00339 & 0.00002 \\
\hline jump $p_{t-1}$ & 0.77113 & 0.01017 \\
\hline \multicolumn{3}{|c|}{$\tau=0.9$} \\
\hline$f p c_{t-1}$ & 1.44195 & 0.00000 \\
\hline$\overline{f p c_{5 t-1}}$ & 0.15027 & 0.52722 \\
\hline$v_{i x-1}$ & -0.00002 & 0.87801 \\
\hline$s p 500_{t-1}$ & -0.00919 & 0.00000 \\
\hline jumpt-1 & -0.07240 & 0.90576 \\
\hline
\end{tabular}

The table reports the coefficients and the $p$-values for Model (5); $\tau=\{0.1,0.5,0.9\}$. The standard errors are computed by means of the bootstrapping procedure, by employing the $x y$-pair method.

Table 2. Restricted model against unrestricted model.

\begin{tabular}{cccc}
\hline \multirow{2}{*}{$\boldsymbol{c}$} & $\boldsymbol{R}^{\mathbf{1}}(\boldsymbol{\tau})$ & $\boldsymbol{R}^{\mathbf{1}}(\boldsymbol{\tau})$ & $\boldsymbol{\xi}_{\boldsymbol{w}}$ \\
\cline { 2 - 4 } & Unrestricted Model & Restricted Model & $\boldsymbol{p}$-Value \\
\hline 0.1 & 0.3655 & 0.2940 & 0.0000 \\
0.2 & 0.4439 & 0.3785 & 0.0000 \\
0.3 & 0.5005 & 0.4422 & 0.0000 \\
0.4 & 0.5427 & 0.4952 & 0.0000 \\
0.5 & 0.5801 & 0.5396 & 0.0000 \\
0.6 & 0.6142 & 0.5803 & 0.0000 \\
0.7 & 0.6474 & 0.6223 & 0.0000 \\
0.8 & 0.6865 & 0.6755 & 0.0007 \\
0.9 & 0.7492 & 0.7467 & 0.9123 \\
\hline
\end{tabular}

The table reports some results coming from the comparison between the unrestricted model, that is Model (5), and the restricted model, in which the regressors are just $f p c_{t-1}$ and $s p 500_{t-1}$. The second and the third columns give the pseudo coefficients of determination, whereas the fourth one gives the $p$-values of the test statistic introduced by Koenker and Bassett [51]. 
Returning to the coefficients' values, we note that the impact on the FPC conditional quantiles of $f p c_{t-1},{\overline{f p c_{5}-1}}_{t}, v i x_{t-1}$ and $j u m p_{t-1}$ is positive in all cases in which the coefficients are statistically significant. Therefore, there is a positive relationship between these variables and $Q_{f p c_{t}}\left(\tau \mid \mathbf{x}_{t-1}\right)$. With respect to the price jumps, the positive impact is a somewhat expected result, and it extends the findings in Corsi et al. [52] about the impact of price jumps on realized volatility. However, as the coefficient of $s p 500_{t-1}, \hat{\beta}_{4}(\tau)$, is always negative, in keeping with Black [53], we find that an increasing market return implies greater stability and negative effects on $Q_{f p c_{t}}\left(\tau \mid \mathbf{x}_{t-1}\right)$. We also checked on the persistence of volatility, measured by the sum of the HAR coefficients, that is, $\hat{\beta}_{1}(\tau)+\hat{\beta}_{2}(\tau)$, and noted that persistence is stronger at high levels of $\tau: \hat{\beta}_{1}(0.1)+\hat{\beta}_{2}(0.1)=0.396, \hat{\beta}_{1}(0.5)+\hat{\beta}_{2}(0.5)=0.734$ and $\hat{\beta}_{1}(0.9)+\hat{\beta}_{2}(0.9)=1.5923$. This evidence, which is coherent with the result for jumps, suggests that volatility in high regimes (upper quantiles) is more persistent as opposed to median or low regimes (lower quantiles) and that unexpected movements/shocks (including jumps) may have a larger effect on lower volatility quantiles compared to their impact on higher volatility quantiles, as they convey relevant information. While these results, recovered from a full-sample analysis, provide an interesting interpretation, they do not take into account the possible structural changes in the relationship between covariates and volatility conditional quantiles. This problem is analysed below.

Even if the coefficients' signs do not change over $\tau$, it is important to determine whether changes in $\tau$ affect their magnitude. In other words, we want to check the so-called location-shift hypothesis, which states that the parameters in the conditional quantile equation are identical over $\tau$. Important information can be drawn from Figure 1, which shows the coefficients' plots.
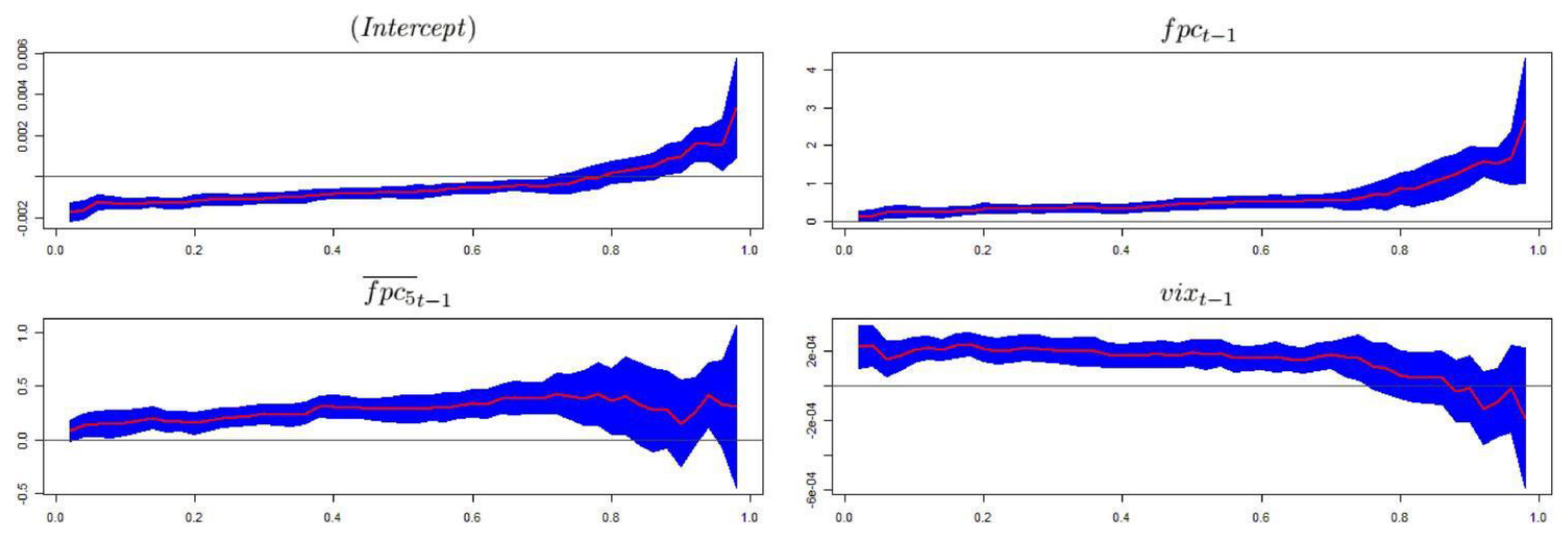

$s p 500_{t-1}$
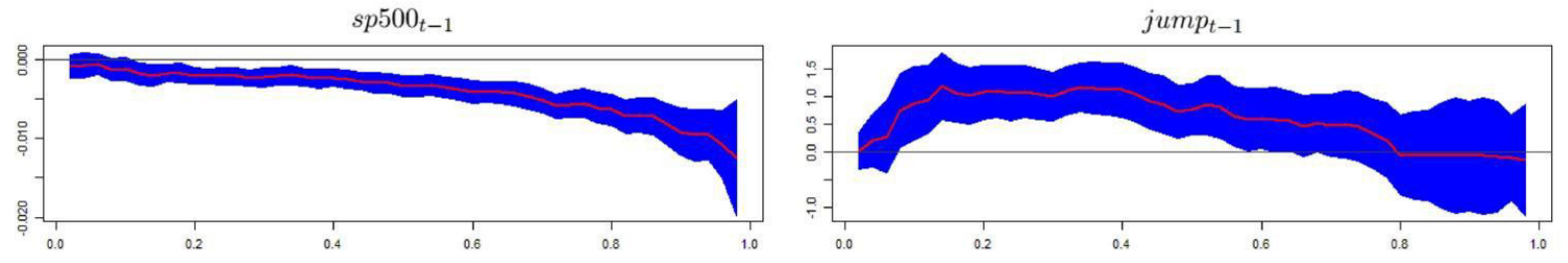

Figure 1. Coefficients plots. The red lines represent the coefficients values over $\tau$ levels, while the blue areas are the associated $95 \%$ confidence intervals.

The impact of $f p c_{t-1}$ on the conditional quantiles of volatility is constant up to $\tau=0.7$, where it increases significantly. In the case of ${\overline{f p c_{5}}}_{t-1}$, we observe a slightly increasing trend until $\tau=0.7$, when the uncertainty level becomes noticeable. The impact of vix $x_{t-1}$ has a flat trend up to $\tau=0.7$, when it begins a decreasing trend, reaching negative values in a region where the regressor is not significant. However, $s p 500_{t-1}$ shows a negative effect on the volatility quantiles, and this relationship grows quickly at high values of $\tau$. We associate this finding with the so-called leverage effect, as argued by Black [53]: increases in volatility are larger when previous returns are negative than when they have the same magnitude but are positive. To verify this claim, we divided the $f p c_{t}$ series into deciles and, conditional to those deciles, computed the mean of $s p 500_{t-1}$ for the various groups. As expected, 
the mean of $s p 500_{t-1}$, corresponding to the values of $f p c_{t}$ in the first decile, is $0.19 \%$, whereas the mean corresponding to the last decile, in which we have the highest $f p c_{t}$ values, is $-0.28 \%$. As a consequence, the negative coefficients for $s p 500_{t-1}$ can be seen as supporting the existence of the leverage effect. Finally, in the case of $j u m p_{t-1}$, we observe a wide band, where its impact grows at the beginning but takes negative turns from $\tau=0.4$.

To summarize, we verify that the relationships between the regressors and the response variable are not constant over $\tau$. In particular, the impact of $f p c_{t-1}$ and $s p 500_{t-1}$ grows considerably at high values of $\tau$. Therefore, $f p c_{t-1}$ and $s p 500_{t-1}$ are critical indicators in the context of extreme events where volatility can reach high levels. The coefficients of the other explanatory variables do not exhibit particular trends at low-medium levels of $\tau$, and when $\tau$ assumes high values, they become even more volatile in a region of high uncertainty, given their wide confidence bands.

An analysis of the coefficients' plots shown in Figure 1 suggests that the hypothesis of equal slopes does not hold. To reach more accurate conclusions, we perform a variant of the Wald test introduced by Koenker and Bassett [54]. The null hypothesis of the test is that the coefficient slopes are the same across quantiles. The test is performed taking into account three distant values of $\tau$, $\tau=\{0.1,0.5,0.9\}$, to cover a wide interval. When we compare the models estimated for $\tau=0.1$ and $\tau=0.5$, the null hypothesis is rejected at the $95 \%$ confidence level for $f p c_{t-1},{\overline{f p c_{5}}}_{t-1}$ and $s p 500_{t-1}$. When we consider $\tau=0.5$ and $\tau=0.9$, the null hypothesis is rejected at the $99 \%$ confidence level for $f p c_{t-1}$ and $s p 500_{t-1}$. We obtain the same result when we focus on $\tau=0.1$ and $\tau=0.9$, so we have evidence against the location-shift hypothesis for those regressors. This finding confirms that the relationship between covariates and conditional quantiles varies across quantile values. This fundamentally relevant finding highlights that when the interest lies in specific volatility quantiles, linear models can lead to inappropriate conclusions about whether there is a relationship between covariates and volatility measures, and if there is, about the strength of the relationship.

\subsection{Rolling Analysis}

The U.S. subprime crisis and the European sovereign debt crisis have had noticeable effects on the financial system, with possible impacts also on the relationship between volatility and its determinants. Therefore, it is important to determine whether these events also affect the parameters of Model (5). To this end, we perform a rolling analysis with steps of one day using a window size of 500 observations and $\tau$ ranging from 0.05 to 0.95 with steps of 0.05 . Therefore, we consider 19 levels of $\tau$ and, for a given $\tau$, obtain 2133 estimates of a single coefficient. The finer grid adopted here allows us to recover a more accurate picture of the evolution of conditional quantiles. Nevertheless, the most relevant quantiles in this case are the upper quantiles, that are associated with the highest volatility levels. The estimated coefficients across time and quantiles are summarized in several figures.

Figure 2 shows the evolution of the relationship between $f p c_{t-1}$ and the conditional volatility quantiles over time and over $\tau$. The first result that arises from Figure 2 is that the impact of $f p c_{t-1}$ has a comparatively stable trend over time for medium-low $\tau$ levels; some jumps are recorded, mainly in the period of the subprime crisis, but their magnitude is negligible. The picture significantly changes in the region of high $\tau$ levels, where the surface is relatively flat and lies at low values in the beginning. However, after the second half of 2007, when the effects of the subprime crisis start to be felt, there is a clear increase in the coefficient values, which reach their peak in the months between late 2008 and the beginning of 2009. Moreover, in this period, we record the highest volatilities in the $f p c_{t-1}$ coefficients over $\tau$ levels. In the following months, the coefficient values decrease, but they remain at high levels until the end of the sample period. $f p c_{t-1}$ is a highly relevant variable for explaining the entire conditional distribution of $f p c_{t}$ because it is statistically significant over a large number of quantiles. Figure 2 verifies that the relationship between $f p c_{t}$ and $f p c_{t-1}$ is affected by particular events, such as the subprime crisis, mainly at medium-high $\tau$ levels. This finding confirms the change in the parameter across $\tau$ values, with an increasing pattern in $\tau$ and highlights that, during periods of market turbulence where the volatility stays at high levels, the volatility density overreacts to past 
movements of volatility, as the $f p c_{t-1}$ coefficient is larger than one for upper quantiles. Therefore, after a sudden increase in volatility at, say, time $t$, we have an increase in the conditional quantiles for time $t+1$ and therefore an increase in the likelihood that we will observe additional volatility spikes (that is, volatility that exceeds a time-invariant threshold) at time $t+1$.

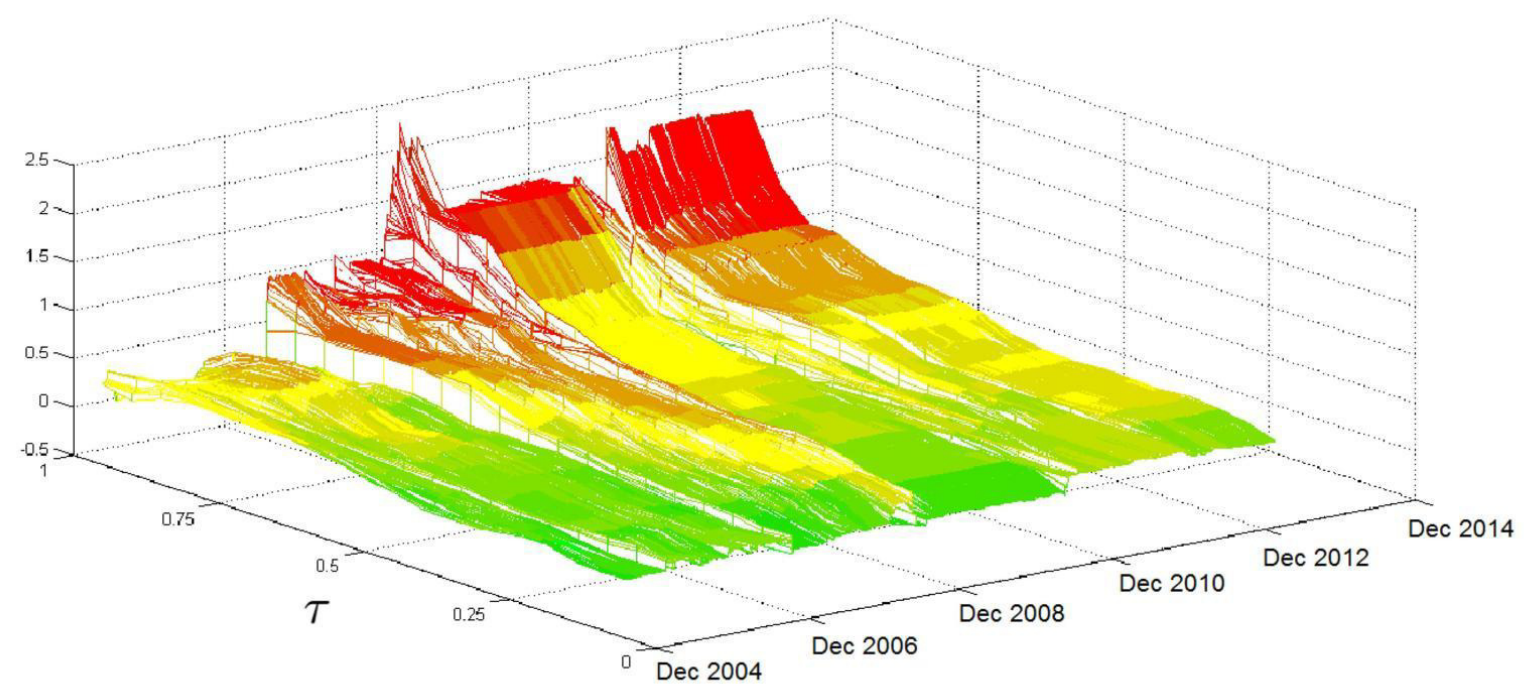

Figure 2. Rolling analysis for $f p c_{t-1}$.

Referring to $\overline{f p c_{5}-1}$, the HAR coefficient reported in Figure 3 has a volatile pattern until late 2008, when it reaches its peak. After that, the surface flattens, but another jump is recorded in mid-2011, mainly in the region of high $\tau$ values. Therefore, the relationship between the $f p c_{t}$ quantiles and $\overline{f p c}_{t-1}$, which reflects the perspectives of investors who have medium time horizons, is volatile over time, mainly in the region of high $\tau$ values. Again, this result can be associated with crises that affect the persistence and the probability that extreme volatilities will occur.

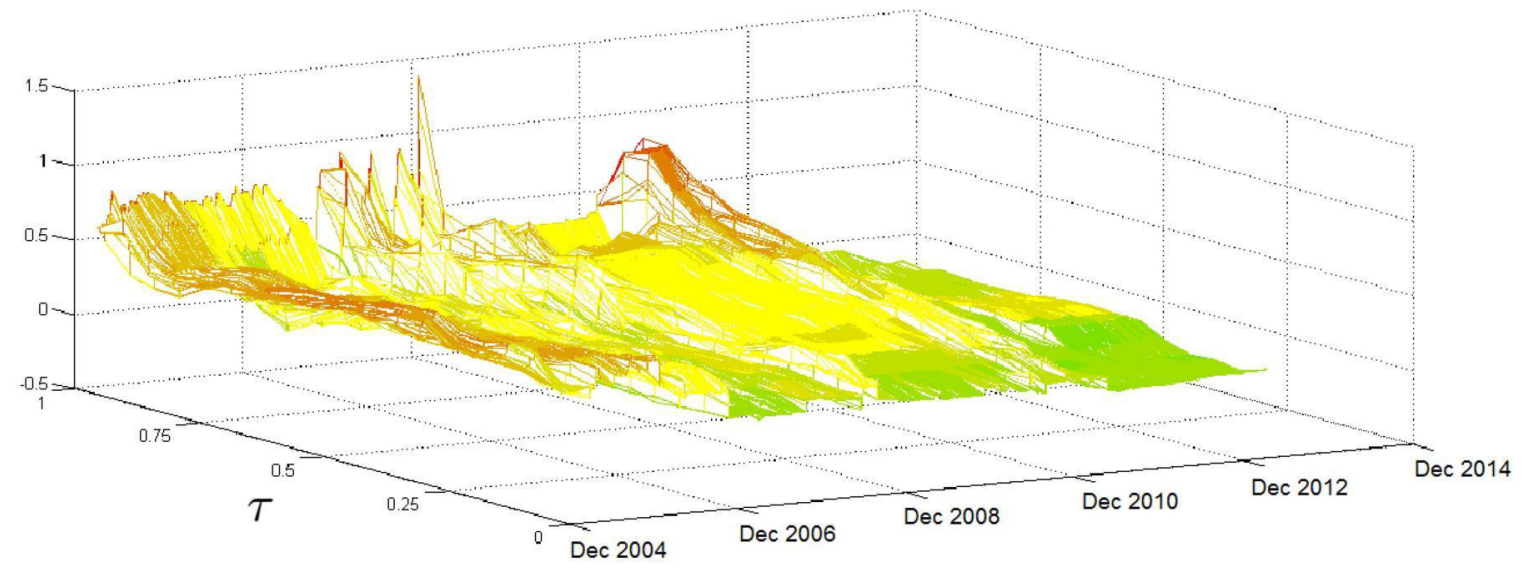

Figure 3. Rolling analysis for ${\overline{f p c_{5}}}_{t-1}$.

Figure 4 shows two periods in which the vix $x_{t-1}$ coefficient has high values: between the end of 2008 and early 2010 and a shorter period from the end of 2011 to the first half of 2012. While, in the first period, the impact of vix $x_{t-1}$ significantly increases for all $\tau$ levels; in the second period, the 
increase in the coefficient affects just the surface region in which $\tau$ takes high values. Unlike the HAR coefficients described above, the vix $x_{t-1}$ coefficient does not have a clear and stable increasing trend over $\tau$. In addition, the relationship between the $f p c_{t}$ conditional quantiles and vix $x_{t-1}$ is highly sensitive to the subprime crisis when pessimism among financial operators, reflected in the implied volatility of the S\&P 500 index options, was acute. This result is again somewhat expected because we focus on U.S.-based data, and the subprime crisis had a high impact on the U.S. equity market. Our results are evidence that the perception of market risk has a great impact on the evolution of market volatility (as proxied by $f p c_{t}$ ), particularly during financial turmoil. The impact is not so clear-cut during the European sovereign crisis, which had less effect on the U.S. equity market.

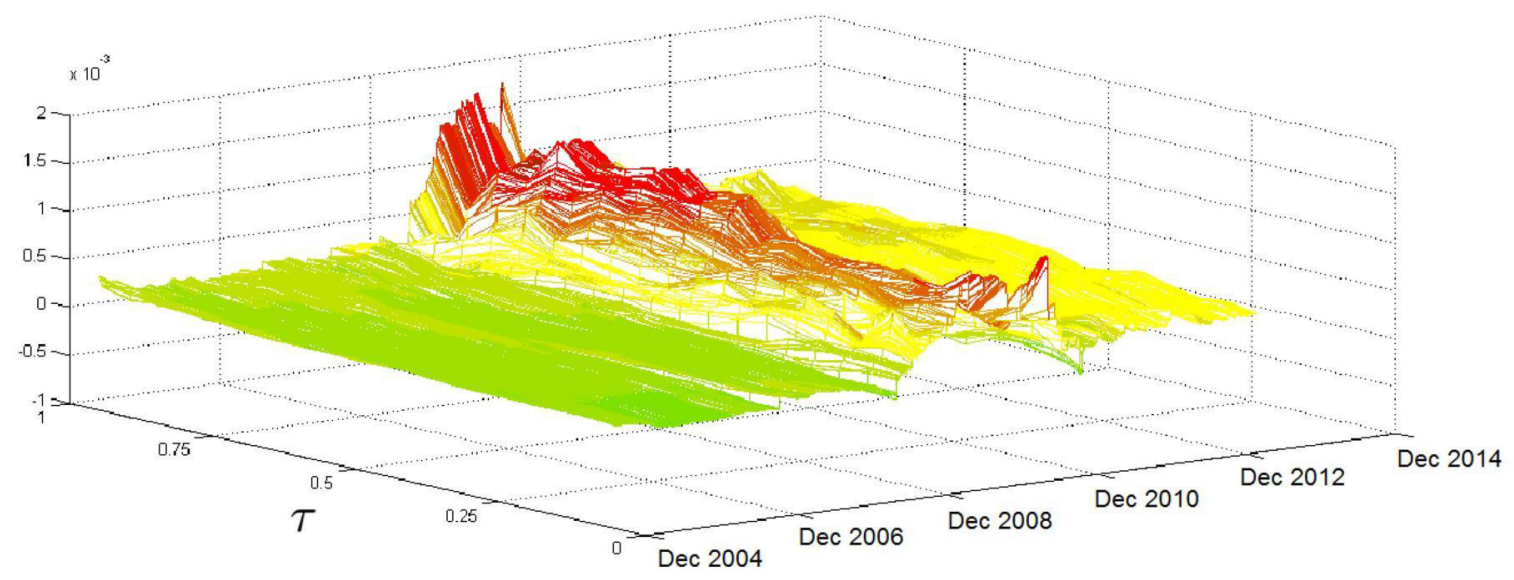

Figure 4. Rolling analysis for vix $x_{t-1}$.

Figure 5 shows how the impact of $s p 500_{t-1}$ evolves over time and over $\tau$. The surface given is almost always flat, the exception being the months between late 2008 and the end of 2010, when the effects of the subprime crisis were particularly acute; during this time, the coefficient values decrease as $\tau$ grows, mainly for values of $\tau$ above the median. The lagged value of the S\&P 500 index return affects the entire conditional distribution of $f p c_{t}$ and is statistically significant in almost all the quantiles considered. Moreover, Figure 5 shows that the effect is negative and particularly pronounced during the subprime crisis when negative returns exacerbated market risk, increasing the upper quantiles' volatility and increasing the likelihood of large and extreme volatility events.

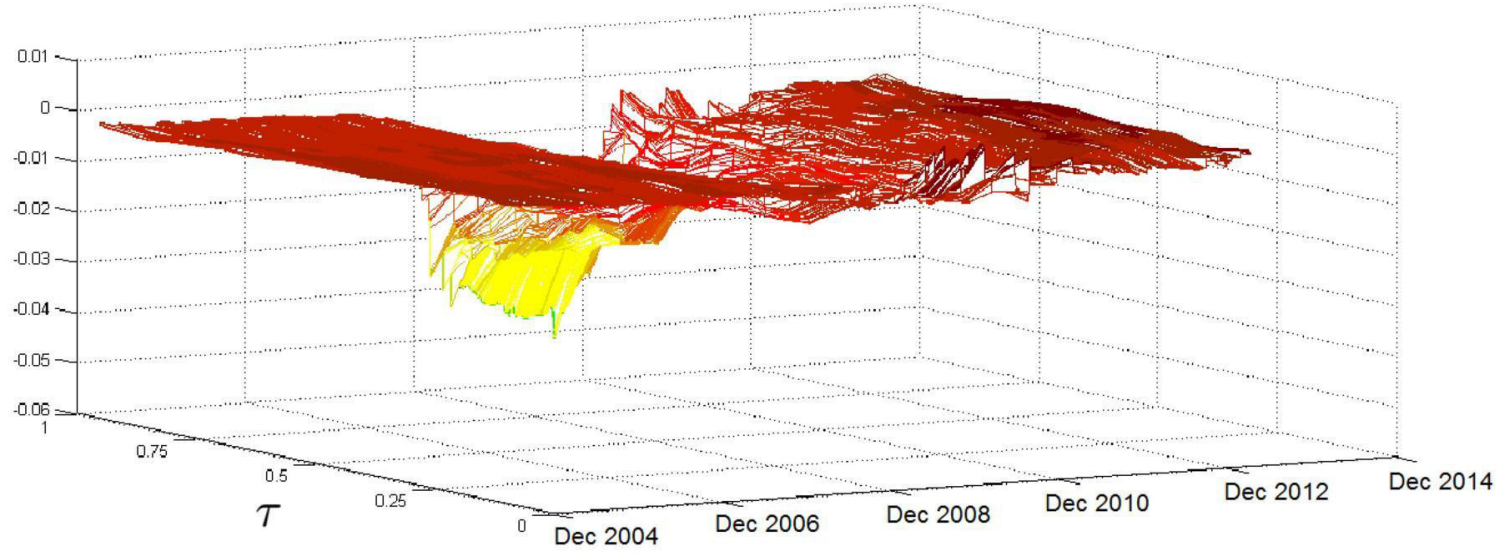

Figure 5. Rolling analysis for $s p 500_{t-1}$. 
Finally, Figure 6 shows the surface associated with the jump component. At the beginning of the sample, the jump $p_{t-1}$ coefficient takes on small values over the $\tau$ levels; however, it starts to grow in 2007, reaching high peaks at high $\tau$ levels during the subprime crisis. Although the coefficient reaches considerable values in this region, their statistical significance is limited. After the second half of 2009, the surface flattens out again until the end of the sample, with the exception of some peaks of moderate size that were recorded in 2011 during the sovereign debt crises.

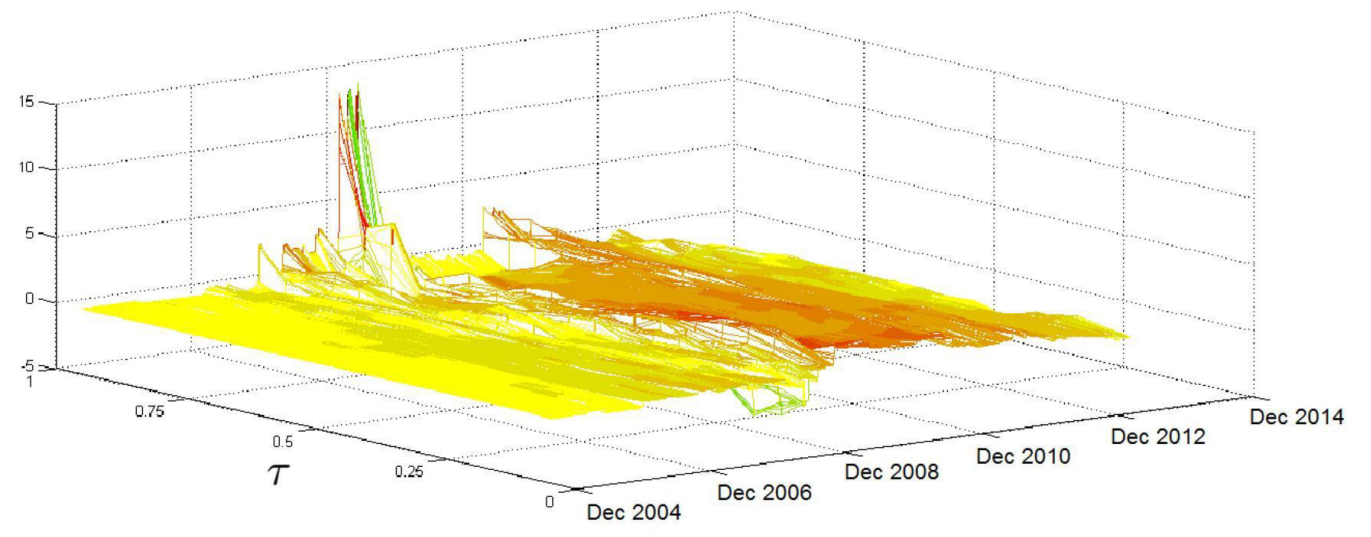

Figure 6. Rolling analysis for jump $p_{t-1}$.

To summarize, using the rolling analysis, we show that two special and extreme market events (the U.S. subprime crisis and the European sovereign debt crisis) affected the relationships between the realized volatility quantiles and a set of covariates. Our results show that coefficients can reasonably vary, with a potential and relevant impact on the forecasts of both the mean (or median) volatility and the volatility distribution (starting from the quantiles). The effects differ across quantiles and change with respect to the volatility upper tails, as compared to the median and the lower tail. Therefore, when volatility quantiles are modeled, the impacts of covariates might differ over time and over quantiles, being crucial during certain market phases. This result further supports the need for quantile-specific estimations when there is an interest in single volatility quantiles.

\subsection{Evaluation of the Predictive Power}

We evaluate the volatility density forecasts using the tests of Berkowitz [32], Amisano and Giacomini [33] and Diebold and Mariano [34]. We carry out the three tests by estimating a larger number of $f p c_{t}$ conditional quantiles with respect to those analysed in Section 5.2. This allows recovering more precise volatility distribution functions. In particular, we consider 49 values of $\tau$, ranging from 0.02 to 0.98 , with steps of 0.02 . Given the findings of the previous subsection, we must use a rolling procedure to build density forecasts. However, we modify the rolling scheme previously adopted to keep a balance between the reliability of the estimated coefficients and computational times. In particular, we recover the forecasts based on conditional quantiles estimated from subsamples of 100 observations, and we roll over the sample every 10 days. Within each 10-day window, we use fixed coefficients but produce one step-ahead forecasts updating the conditioning information set.

With regard to the Berkowitz test, in the case of Model (5), we checked that $z_{t}$ is normally distributed, as the likelihood ratio test $L R_{b}$ equals 5.26 and the null hypothesis of the Berkowitz test, that is, $z_{t} \sim \mathcal{N}(0,1)$ with no autocorrelation, is not rejected at the $5 \%$ significance level, thus validating the forecast goodness of Model (5). To determine whether the predictive power of our approach is affected by the U.S. subprime and the European sovereign debt crises, the series $z_{t}$ is divided into two parts of equal length: the first referring to a period of relative calm, from the beginning of 2003 to the first half of 2007, and the second referring to a period of market turmoil that was due to the two crises 
between the second half of 2007 and the first half of 2013. In the first part, $L R_{b}$ equals 2.34 , and in the second it equals 5.39. Nevertheless, the null hypothesis of the Berkowitz test is not rejected at the $5 \%$ level in both cases. Therefore, the conditional quantile model and the approach that we adopt to recover the conditional density forecasts are appropriate even during financial turbulence. An analysis of the results reveals that, as in the analysis of the full sample, $\overline{f p c_{5}}{ }_{t-1}$, vix $x_{t-1}$ and jump $p_{t-1}$ are not significant to explain the volatility quantiles at high values of $\tau$ in many of the subsamples. Therefore, we must determine whether this result affects the output of the Berkowitz test using a restricted model in which the regressors are only $f p c_{t-1}$ and $s p 500_{t-1} . L R_{b}$ equals 2.60 , a smaller value than in the previous cases. The last findings reported above suggest that the inclusion of non-significant explanatory variables penalizes the predictive power of Model (5). The restricted model gives the lowest value of $L R_{b}$, but the predictive power could be improved by selecting only those variables that are significant for each value of $\tau$ and for each subsample to forecast the conditional distribution of the volatility. Thus, the structure of Model (5) would change over time and over $\tau$. However, this approach is not applied in the present work because it would require using only the significant variables in 49 models, one for each specific value of $\tau$, while it should be considered across all the rolling subsamples.

So far, we have focused on an absolute assessment of our approach. Now, we compare it with a competing model that is fully parametric. We recover the predictive conditional distribution of the realized range volatility using a HARX model in which the mean dynamic is driven by a linear combination of the explanatory variables $f p c_{t-1}$ and ${\overline{f p c_{5}}}_{t-1}$ (the HAR terms) and the exogenous variables vix $x_{t-1}, s p 500_{t-1}$ and jump $p_{t-1}$ (the $\mathrm{X}$ in the model's acronym). In addition, to capture the volatility-of-volatility effect argued by Corsi et al. [31], a GJR-GARCH term [30] is introduced to the innovation (we name the model HARX-GJR). The error term is also assumed to follow a normal-inverse Gaussian $(\mathcal{N} \mathcal{I} \mathcal{G})$ distribution with a mean of 0 and a variance of 1 . Thus, the conditional variance is allowed to change over time, and the distribution of the error is flexible in relation to features like fat tails and skewness. We start by using the Berkowitz test to evaluate the density forecast performance of the HARX-GJR model. The likelihood ratio test $L R_{b}$ equals 113.88 , a high value that suggests a clear rejection of the null hypothesis. We also determined whether the HARX-GJR model works better when we use the logarithm of the volatility as a response variable, following the evidence in Corsi et al. [52], and found that the likelihood ratio test $L R_{b}$ provides a much lower value (20.27). Even so, the test signals a rejection of the null hypothesis with a low $p$-value. As a first finding, our approach provides more flexibility than the parametric HARX-GJR model does and is better in terms of the Berkowitz test.

To provide more accurate results, we move to a comparison of our approach to the HARX-GJR using the Amisano and Giacomini [33] and the Diebold and Mariano [34] tests. With regard to the Amisano and Giacomini [33] test, we use four weights to compute the quantity given in Equation (12): $w_{C E}\left(f p c_{t}^{s t}\right), w_{R T}\left(f p c_{t}^{s t}\right), w_{L T}\left(f p c_{t}^{s t}\right)$ and $w_{N W}\left(f p c_{t}^{s t}\right)$. The associated likelihood ratio tests are denoted by $A G_{C E}, A G_{R T}, A G_{L T}$ and $A G_{N W}$, respectively. Overall, our approach provides better results than the HARX-GJR because $\overline{W L R}$ is always positive; in fact, $A G_{C E}=1.6521, A G_{R T}=2.2766$, $A G_{L T}=1.2961$ and $A G_{N W}=1.8810$. Given that the critical values at the $5 \%$ level are equal to -1.96 and 1.96 (we are dealing with a two-sided test), the null hypothesis of equal performance is rejected only when we give a higher weight to the right tail of the volatility conditional distribution.

Similar results are obtained when we consider the single quantile loss function and the Diebold and Mariano [34] test statistic. Our approach provides lower losses because $\overline{d_{D M, \tau}}$ is negative for all the $\tau$ levels we considered $(0.1,0.5,0.9)$. However, the differences are statistically significant only at $\tau=0.9$, given that $D M_{0.1}=-1.7283$ (0.0839), $D M_{0.5}=-1.7332$ (0.0831) and $D M_{0.9}=-2.6884$ (0.0072).

To summarize, the results demonstrate the good performance of our approach, in particular when we focus on the right tail of the FPC (a kind of market risk factor) distribution. We note that the right tail assumes critical importance in our framework, as it represents periods of extreme risks. Our findings indicate another relevant contribution of this study, as the quantile regression approach that we propose can be used to recover density forecasts for a realized volatility measure. These forecasts 
improve on those of a traditional approach because of the inclusion of quantile-specific coefficients. This feature of our approach might become particularly relevant in all empirical applications where predictive volatility density is required.

\subsection{Single-Asset Results}

The results in Sections 5.1-5.3 were based on a summary of the 16 asset volatility movements, which was itself based on the FPC. Now, we search for confirmation of the main findings of Model (5) by running Model (6) at the single-asset level for all 16 assets. For simplicity, only the results associated with $\tau=\{0.1,0.5,0.9\}$ are shown (additional tables and figures relating to the single-asset estimates are available on request). Table 3 provides the estimated parameters and their $p$-values. We first focus on the relationships between $Q_{r r v_{i, t}}\left(\tau \mid \mathbf{x}_{i, t-1}\right)$ and $r r v_{i, t-1}$, for $i=1, \ldots, 16$. When $\tau$ equals $0.1, r r v_{i, t-1}$ is not significant at the $5 \%$ level to explain the conditional volatility quantiles of eight assets: $A T T, C A T$, $H O N, I B M, P E P, P R G, T W X$ and TXN. At $\tau=0.5, r r v_{i, t-1}$ is not significant only for $P R G$, whereas, at $\tau=0.9, \operatorname{rrv}_{i, t-1}$ is not significant for PEP and PRG. Compared with the other regressors and in line with the results obtained for the FPC, $r r v_{i, t-1}$ is one of the most significant explanatory variables at high levels of $\tau$, so it assumes critical importance in the context of extreme events. The $r r v_{i, t-1}$ coefficient takes a negative value only for PEP at $\tau=0.1$, but here it is not statistically significant. In all the other cases, it is always positive. Moreover, the magnitude of the impact that $r r v_{i, t-1}$ has on $Q_{r r v_{i, t}}\left(\tau \mid \mathbf{x}_{i, t-1}\right)$ is a positive function of $\tau$, for all 16 assets.

The differences among the assets increase as $\tau$ grows, and at $\tau=0.9$ the financial companies (BAC, CTG, JPM and WFC) record the highest coefficient values, highlighting the crucial importance of the extreme events in the financial system. $f p c_{t-1}$, the homologous regressor included in Model (5), has a weaker impact on the conditional volatility quantiles than $r r v_{i, t-1}$ does only for the financial companies BAC $(\tau=0.1), J P M(\tau=0.5)$ and CTG $(\tau=0.9)$. Therefore, the relationships between the conditional volatility quantiles and the lagged value of the response variable are stronger for the FPC than for the single assets. $\overline{r r v}_{5 i, t-1}$ is not significant (significance level of 0.05 ) at $\tau=0.1$ for $C T G$, $J P M$ and $P R G$, as the $p$-values of its coefficient indicate. At $\tau=0.5$ it is not significant only for $P R G$, whereas when $\tau$ equals 0.9 it is not significant for CTG, JPM and PRG. The $\overline{r r v}_{5 i, t-1}$ coefficient is always positive and, with the exception of CTG and JPM, it is a positive function of $\tau$. Moreover, the differences among the $\overline{r r v_{5}} i, t-1$ coefficient values are more marked at high $\tau$ levels for all 16 assets. As for the comparison with the results obtained from Model (5), at $\tau=\{0.1,0.5\}, \overline{r r v}_{5}$,t-1 has a stronger impact on the conditional volatility quantiles with respect to ${\overline{f p c_{5}}}_{t-1}$ for most of the 16 assets. When $\tau$ equals 0.9 , it is pointless to compare the coefficients' values because ${\overline{f p c_{5}}}_{t-1}$ is not statistically significant at that level. Therefore, when we take into account the explanatory variables ${\overline{f p c_{5}}}_{t-1}$ and $\overline{r r v}_{5, t-1}$, we record stronger relationships for Model (6) than for Model (5).

The $p$-values of the vix $x_{t-1}$ coefficient are less than 0.05 at $\tau=\{0.1,0.5\}$ for all 16 assets. When $\tau$ equals 0.9 , vix $x_{t-1}$ is significant in the cases of $H O N, H P Q, P R G, T W X$ and TXN. Unlike the coefficients previously mentioned, that of $v i x_{t-1}$ does not have a particular trend over $\tau$; it takes positive values for all 16 assets, therefore, as in the context of the FPC, it has a positive impact on the conditional volatility quantiles. In addition, at $\tau=\{0.1,0.5\}$, the coefficient of vix $x_{t-1}$ is larger in Model (5) than it is in Model (6) for all 16 companies. Therefore, vix $x_{t-1}$ has a more marked impact on the conditional volatility quantiles of the FPC. The comparisons made at $\tau=0.9$ are useless given the high $p$-values of the coefficients of interest.

$s p 500_{t-1}$ is always significant $(\alpha=0.05)$ for the 16 assets, with the exception of BAC, CTG and $J P M$ at $\tau=0.1$. Its coefficient is always negative, as expected, so $s p 500_{t-1}$ has a negative impact on the conditional volatilities' quantiles. In addition, the magnitude of the impact is a negative function of $\tau$ for all the assets, and those relationships become more marked at high $\tau$ levels. With the exception of one case (TXN at $\tau=0.1$ ), the impact of $s p 500_{t-1}$ at $\tau=\{0.1,0.5,0.9\}$ is more pronounced for the conditional volatility quantiles of the FPC than it is when the assets are considered individually, as comparing the absolute values of the related coefficients shows. 
Table 3. Quantile regression results for each asset.

\begin{tabular}{|c|c|c|c|c|c|c|}
\hline Stocks & Intercept & $r r v_{i, t-1}$ & $\overline{r r v}_{5} i, t-1$ & $v_{i x} x_{t-1}$ & $s p 500_{t-1}$ & $j u m p_{i, t-1}$ \\
\hline \multicolumn{7}{|c|}{$\tau=0.1$} \\
\hline ATT & $-0.002(11.24)$ & 4.589 (70.99) & $28.691(0.12)$ & $0.002(0.27)$ & $0.055(0.09)$ & $3.515(21.81)$ \\
\hline BAC & $-0.012(0.02)$ & $25.007(0.00)$ & 14.869 (1.09) & $0.005(0.01)$ & $-0.033(18.18)$ & $-0.202(99.31)$ \\
\hline BOI & $-0.008(0.09)$ & $15.852(0.96)$ & $21.132(0.01)$ & $0.004(0.01)$ & $-0.081(0.01)$ & 4.509 (37.15) \\
\hline CAT & $-0.012(0.00)$ & $10.649(19.31)$ & $25.142(0.00)$ & $0.006(0.00)$ & $-0.067(0.695)$ & $-0.001(99.97)$ \\
\hline CTG & $-0.030(0.00)$ & $18.252(3.74)$ & $12.645(8.61)$ & $0.012(0.00)$ & $-0.081(8.24)$ & $95.128(0.09)$ \\
\hline FDX & $-0.013(0.00)$ & $18.287(0.46)$ & $23.422(0.00)$ & $0.006(0.00)$ & $-0.065(0.34)$ & -7.955 (11.99) \\
\hline $\mathrm{HON}$ & $-0.012(0.00)$ & $7.855(36.51)$ & $26.336(0.01)$ & $0.005(0.00)$ & $-0.097(0.05)$ & $1.054(70.67)$ \\
\hline HPQ & $-0.004(1.75)$ & $16.799(0.85)$ & $15.327(0.33)$ & $0.003(0.00)$ & $-0.082(0.21)$ & $-0.039(99.12)$ \\
\hline IBM & $-0.003(0.09)$ & 13.709 (19.22) & $22.922(0.09)$ & $0.002(0.01)$ & $-0.050(0.39)$ & $23.369(3.12)$ \\
\hline $\mathrm{JPM}$ & $-0.019(0.02)$ & $23.775(2.59)$ & $14.460(10.22)$ & $0.008(0.01)$ & $-0.047(13.31)$ & $2.273(89.03)$ \\
\hline MDZ & $-0.005(0.00)$ & $8.280(1.17)$ & $19.490(0.00)$ & $0.002(0.00)$ & $-0.027(0.49)$ & $-0.297(82.09)$ \\
\hline PEP & $-0.005(0.03)$ & $-2.564(76.78)$ & $26.404(0.00)$ & $0.003(0.00)$ & $-0.036(6.06)$ & $6.751(32.14)$ \\
\hline PRG & $-0.006(0.08)$ & $1.883(86.89)$ & $0.483(91.48)$ & $0.003(0.00)$ & $-0.025(0.72)$ & $10.243(49.12)$ \\
\hline TWX & $-0.005(1.15)$ & 13.005 (19.93) & $29.034(0.00)$ & $0.003(0.16)$ & $-0.072(0.03)$ & $18.752(0.85)$ \\
\hline TXN & $-0.003(19.32)$ & $9.522(16.87)$ & $32.663(0.00)$ & $0.002(0.38)$ & $-0.137(0.00)$ & $0.907(82.14)$ \\
\hline WFC & $-0.012(0.00)$ & $20.878(0.269)$ & $20.321(0.02)$ & $0.005(0.00)$ & $-0.064(0.25)$ & $-0.044(99.77)$ \\
\hline \multicolumn{7}{|c|}{$\tau=0.5$} \\
\hline ATT & $-0.002(12.24)$ & $30.163(0.67)$ & $52.921(0.00)$ & $0.001(4.07)$ & $-0.079(0.00)$ & $-0.400(91.67)$ \\
\hline BAC & $-0.012(0.19)$ & $38.505(0.00)$ & $40.227(0.00)$ & $0.005(0.14)$ & $-0.167(0.00)$ & $14.596(66.46)$ \\
\hline BOI & $-0.011(0.01)$ & $36.842(0.00)$ & $36.566(0.00)$ & $0.005(0.00)$ & $-0.112(0.00)$ & $7.570(35.86)$ \\
\hline CAT & $-0.013(0.00)$ & $40.863(0.00)$ & $40.806(0.00)$ & $0.005(0.00)$ & $-0.141(0.00)$ & $-0.043(99.25)$ \\
\hline CTG & $-0.031(0.06)$ & $44.363(0.00)$ & $25.602(0.04)$ & $0.013(0.05)$ & $-0.109(0.41)$ & $71.026(2.39)$ \\
\hline FDX & $-0.011(0.00)$ & $33.461(0.00)$ & $44.110(0.00)$ & $0.005(0.00)$ & $-0.133(0.00)$ & $-4.139(11.64)$ \\
\hline $\mathrm{HON}$ & $-0.009(0.01)$ & $33.249(0.02)$ & $42.692(0.00)$ & $0.004(0.00)$ & $-0.126(0.00)$ & $-2.821(34.55)$ \\
\hline $\mathrm{HPQ}$ & $-0.006(0.07)$ & $33.324(0.00)$ & $39.040(0.00)$ & $0.003(0.00)$ & $-0.135(0.00)$ & $-1.217(69.97)$ \\
\hline $\mathrm{IBM}$ & $-0.004(0.14)$ & $33.790(0.01)$ & $42.321(0.00)$ & $0.002(0.03)$ & $-0.084(0.00)$ & $39.259(15.63)$ \\
\hline JPM & $-0.016(0.01)$ & $50.524(0.00)$ & $26.286(0.00)$ & $0.007(0.01)$ & $-0.161(0.00)$ & $-2.738(92.41)$ \\
\hline MDZ & $-0.006(0.00)$ & $30.852(0.00)$ & $36.733(0.00)$ & $0.003(0.00)$ & $-0.050(0.00)$ & $-1.370(70.27)$ \\
\hline PEP & $-0.004(0.01)$ & $31.618(3.74)$ & $39.554(0.57)$ & $0.002(0.00)$ & $-0.047(0.00)$ & $3.569(60.58)$ \\
\hline PRG & $-0.010(0.22)$ & $11.543(65.76)$ & $21.804(20.76)$ & $0.005(0.13)$ & $-0.050(0.55)$ & $120.000(10.11)$ \\
\hline TWX & $-0.007(0.01)$ & $37.437(0.00)$ & $39.468(0.00)$ & $0.003(0.00)$ & $-0.098(0.00)$ & $18.763(2.51)$ \\
\hline $\mathrm{TXN}$ & $-0.002(24.69)$ & $27.555(0.00)$ & $49.860(0.00)$ & $0.002(0.63)$ & $-0.168(0.00)$ & $-0.497(91.84)$ \\
\hline WFC & $-0.011(0.59)$ & $37.207(0.00)$ & $43.713(0.00)$ & $0.005(0.52)$ & $-0.133(0.00)$ & $-0.109(99.18)$ \\
\hline \multicolumn{7}{|c|}{$\tau=0.9$} \\
\hline ATT & $-0.003(32.72)$ & $70.584(0.00)$ & $69.582(0.00)$ & $0.002(14.33)$ & $-0.179(0.00)$ & $0.282(98.09)$ \\
\hline BAC & $-0.005(60.02)$ & $104.077(0.00)$ & $48.227(0.39)$ & $0.002(60.64)$ & $-0.339(0.02)$ & $156.280(21.58)$ \\
\hline BOI & $-0.008(17.55)$ & $60.555(0.01)$ & $79.933(0.00)$ & $0.004(14.54)$ & $-0.254(0.00)$ & $-0.471(97.86)$ \\
\hline CAT & $-0.005(40.02)$ & $79.303(0.00)$ & $61.374(0.00)$ & 0.003 (22.98) & $-0.321(0.00)$ & $-0.103(99.14)$ \\
\hline CTG & $-0.003(86.81)$ & $158.940(0.11)$ & 19.051 (54.87) & 0.001 (83.97) & $-0.376(0.02)$ & $-73.284(15.63)$ \\
\hline FDX & $-0.006(16.06)$ & $57.441(0.00)$ & $78.243(0.00)$ & $0.004(6.12)$ & $-0.290(0.00)$ & $-12.050(44.62)$ \\
\hline $\mathrm{HON}$ & $-0.009(9.87)$ & $69.621(0.00)$ & $60.597(0.00)$ & $0.005(3.83)$ & $-0.321(0.00)$ & $-0.630(92.34)$ \\
\hline $\mathrm{HPQ}$ & $-0.008(12.83)$ & $72.014(0.00)$ & $66.113(0.00)$ & $0.004(4.62)$ & $-0.344(0.00)$ & $-3.320(47.36)$ \\
\hline IBM & $-0.001(84.22)$ & $55.602(2.08)$ & $101.283(0.02)$ & $0.000(89.26)$ & $-0.218(0.00)$ & $41.659(55.83)$ \\
\hline JPM & $-0.014(6.25)$ & $134.345(0.00)$ & $17.516(5.77)$ & $0.006(6.81)$ & $-0.349(0.00)$ & $155.650(8.94)$ \\
\hline MDZ & $0.000(97.15)$ & $86.207(0.00)$ & $53.696(0.18)$ & $0.001(50.69)$ & $-0.155(0.00)$ & $-3.639(23.02)$ \\
\hline PEP & $0.000(91.96)$ & $36.885(36.23)$ & $117.046(2.80)$ & $0.000(86.11)$ & $-0.107(0.28)$ & $-0.578(96.86)$ \\
\hline PRG & $-0.010(2.21)$ & $59.766(16.98)$ & 47.298 (13.89) & $0.004(1.35)$ & $-0.154(0.01)$ & $152.132(37.35)$ \\
\hline TWX & $-0.010(0.48)$ & $86.695(0.00)$ & $52.144(0.00)$ & $0.004(0.42)$ & $-0.232(0.00)$ & -1.217 (94.69) \\
\hline $\mathrm{TXN}$ & $-0.015(0.22)$ & $56.407(0.00)$ & $80.214(0.00)$ & $0.007(0.03)$ & $-0.381(0.00)$ & $4.170(60.83)$ \\
\hline WFC & $-0.004(57.68)$ & $94.470(0.00)$ & $64.679(0.00)$ & $0.002(53.26)$ & $-0.309(0.00)$ & $-0.292(99.59)$ \\
\hline
\end{tabular}

The table reports, for each stock, the coefficients (\%) and the $p$-values (\%) for Model (6), given $\tau=\{0.1,0.5,0.9\}$. 
At $\tau=0.1, j u m p_{i, t-1}$ is significant, at the $5 \%$ level, for CTG, IBM and TWX. It is significant only for $C T G$ and TWX at $\tau=0.5$, whereas it is never significant when $\tau$ equals 0.9 . jump $p_{i, t-1}$ 's coefficient takes both negative and positive values and, with the exception of a few assets, it does not have a particular trend over $\tau$. Comparing these results with those obtained for the $f p c_{t}$ conditional quantiles, we find that, with the exception of CTG $(\tau=0.1)$ and PRG $(\tau=0.5)$, the lagged value of the component associated with jumps has more impact in Model (5) than in Model (6) at $\tau=\{0.1,0.5\}$. It is pointless to compare the coefficients at $\tau=0.9$ given their high $p$-values.

To summarize, the explanatory variables $r r v_{i, t-1}, \overline{r r v}_{5 i, t-1}, v i x_{t-1}$ and $s p 500_{t-1}$ are sufficient to explain the conditional volatility quantiles of the 16 assets in most of the cases studied. Their coefficients tend to take the same sign for the 16 assets: positive in the cases of $r r v_{i, t-1}, \overline{r r v}_{5 i, t-1}$ and vix $x_{t-1}$ and negative in the case of $s p 500_{t-1}$. Moreover, the coefficients of $r r v_{i, t-1}, \overline{r r v}_{5, t-1}$ and $s p 500_{t-1}$ have a clear trend over $\tau$, providing evidence against the location-shift hypothesis, which assumes homogeneous impacts of the regressors across quantiles. However, jump $p_{i, t-1}$ is significant in only a few cases. Furthermore, with the exception of $\overline{r r v}_{5 i, t-1}$, we find that, in most of the studied cases, the relationships between the explanatory variables and the conditional volatility quantiles are more pronounced in the context of the FPC than when the assets are individually considered. This result shows that the FPC captures a kind of systematic effect, where the relationship between macro-level covariates, such as the VIX and the S\&P 500 index, and volatility quantiles is clearer. At the single-asset level, the impact of covariates is more heterogeneous than for the FPC, perhaps suggesting the need for company- (or sector-) specific covariates.

Table 4. Berkowitz test for the single asset analysis.

\begin{tabular}{ccc}
\hline Asset & $\boldsymbol{L R}_{\boldsymbol{b}}$ & $\boldsymbol{L} \boldsymbol{R}_{\boldsymbol{b}, \text { HARX-GJR }}$ \\
\hline ATT & $10.23(0.0167)$ & $150.26(0.0000)$ \\
BAC & $3.00(0.3916)$ & $125.11(0.0000)$ \\
BOI & $7.90(0.04812)$ & $93.09(0.0000)$ \\
CAT & $9.31(0.0254)$ & $88.11(0.0000)$ \\
CTG & $4.28(0.2327)$ & $142.99(0.0000)$ \\
FDX & $12.62(0.0055)$ & $95.63(0.0000)$ \\
HON & $5.95(0.1140)$ & $115.59(0.0000)$ \\
HPQ & $5.33(0.1491)$ & $83.80(0.0000)$ \\
IBM & $4.25(0.2357)$ & $157.06(0.0000)$ \\
JPM & $3.26(0.3532)$ & $124.01(0.0000)$ \\
MDZ & $5.49(0.1392)$ & $159.86(0.0000)$ \\
PEP & $9.97(0.0188)$ & $164.45(0.0000)$ \\
PRG & $2.25(0.5222)$ & $130.88(0.0000)$ \\
TWX & $11.09(0.0112)$ & $99.10(0.0000)$ \\
TXN & $1.90(0.5934)$ & $79.09(0.0000)$ \\
WFC & $7.04(0.0706)$ & $143.04(0.0000)$ \\
\hline
\end{tabular}

The table reports for each stock (the ticker is given in the first column) the values of the likelihood ratio test (the $p$-values are given in brackets) proposed by Berkowitz [32], generated by Model (6), $L R_{b}$, and the HARX-GJR model, $L R_{b, H A R X-G J R}$.

The last point of our analysis refers to the assessment of the predictive power of Model (6), which we apply for each of the 16 assets. As in the case of the model for the FPC, we use the tests Berkowitz [32], Amisano and Giacomini [33] and Diebold and Mariano [34] proposed. With regard to the Berkowitz test, Table 4 provides the values of the likelihood ratio defined by Equation (11) and the results generated by Model (6), showing that the null hypothesis of the test, that is, $z_{t} \sim \mathcal{N}(0,1)$ with no autocorrelation, is not rejected for 10 assets: BAC, CTG, HON, HPQ, IBM, JPM, MDZ, PRG, TXN and WFC. The results from the other six cases stem from the fact that some variables, mainly jump $p_{i, t-1}$, are not significant in many subsamples for several $\tau$ levels. As indicated in Section 5.3, which focused on the FPC, the predictive power of our approach could be improved by selecting for each subsample and each $\tau$ only the regressors that are significant in order to explain the individually evaluated 
conditional quantiles. Thus, the structure of Model (6) would change over time. Now, we compare our approach with the HARX-GJR model. The results that arise from using the HARX-GJR model are given in Table 4 . As in the FPC context, the likelihood ratio test (11), denoted by $L R_{b, H A R X-G J R}$, takes high values for all 16 assets, suggesting that the null hypothesis is rejected with low $p$-values.

Table 5 reports the results of the Amisano and Giacomini [33] test and shows that overall our model provides better results: the test statistic values (13) are in most cases positive, and the null hypothesis of equal performance is almost always rejected at the $5 \%$ level. Similar results are obtained for the Diebold and Mariano [34] test, as we can see from Table 6.

Table 5. Amisano-Giacomini test for the single asset analysis.

\begin{tabular}{ccccc}
\hline Asset & $A G_{N W}$ & $A G_{C E}$ & $A G_{R T}$ & $A G_{L T}$ \\
\hline ATT & $3.8957(0.0001)$ & $4.3336(0.0000)$ & $2.8878(0.0039)$ & $4.6406(0.0000)$ \\
BAC & $4.0325(0.0001)$ & $4.3822(0.0000)$ & $3.0110(0.0026)$ & $5.4706(0.0000)$ \\
BOI & $13.9648(0.0000)$ & $9.3975(0.0000)$ & $18.9700(0.0000)$ & $9.4658(0.0000)$ \\
CAT & $4.3298(0.0000)$ & $4.5230(0.0000)$ & $2.9384(0.0033)$ & $5.1361(0.0000)$ \\
CTG & $3.6318(0.0003)$ & $3.7586(0.0002)$ & $2.8238(0.0047)$ & $4.1108(0.0000)$ \\
FDX & $3.0102(0.0026)$ & $2.9183(0.0035)$ & $3.9842(0.0001)$ & $2.0103(0.0444)$ \\
HON & $2.9055(0.0037)$ & $3.0381(0.0024)$ & $2.4728(0.0134)$ & $2.8784(0.0040)$ \\
HPQ & $16.6222(0.0000)$ & $7.4219(0.0000)$ & $2.7958(0.0052)$ & $5.3210(0.0000)$ \\
IBM & $-0.2210(0.8251)$ & $0.3105(0.7562)$ & $-0.6036(0.5461)$ & $0.5044(0.6140)$ \\
JPM & $0.7630(0.4455)$ & $0.6326(0.5270)$ & $1.0247(0.3055)$ & $0.4642(0.6425)$ \\
MDZ & $-0.3915(0.6954)$ & $-0.3848(0.7004)$ & $-0.3545(0.7230)$ & $-0.4181(0.6759)$ \\
PEP & $1.0614(0.2885)$ & $2.1311(0.0331)$ & $0.0232(0.9815)$ & $2.8849(0.0039)$ \\
PRG & $3.2625(0.0011)$ & $3.2615(0.0011)$ & $3.3250(0.0009)$ & $3.2064(0.0013)$ \\
TWX & $0.4999(0.6171)$ & $0.0756(0.9397)$ & $1.7995(0.0719)$ & $0.1434(0.8860)$ \\
TXN & $3.1661(0.0015)$ & $3.1672(0.0015)$ & $2.4961(0.0126)$ & $3.0988(0.0019)$ \\
WFC & $3.0723(0.0021)$ & $3.1620(0.0016)$ & $2.4755(0.0133)$ & $3.2976(0.0010)$ \\
\hline
\end{tabular}

The table reports, for each stock (the ticker is given in the first column), the values of the likelihood ratio test (the $p$-values are given in brackets) proposed by Amisano and Giacomini [33], for different weights. Each weight places greater emphasis on particular regions of the distribution: center $\left(A G_{C E}\right)$, right tail $\left(A G_{R T}\right)$ and left tail $\left(A G_{L T}\right)$. $A G_{N W}$ coincides with the unweighted likelihood ratio test.

Table 6. Diebold-Mariano test for the single asset analysis.

\begin{tabular}{cccc}
\hline Asset & $\boldsymbol{D M}_{\mathbf{0 . 1}}$ & $\mathbf{D M}_{\mathbf{0 . 5}}$ & $\mathbf{D M}_{\mathbf{0 . 9}}$ \\
\hline ATT & $-1.9227(0.0545)$ & $-2.9818(0.0029)$ & $-3.0612(0.0022)$ \\
BAC & $-1.6373(0.1016)$ & $-1.9346(0.0530)$ & $-1.8357(0.0664)$ \\
BOI & $-1.8368(0.0662)$ & $-3.2551(0.0011)$ & $-3.3817(0.0007)$ \\
CAT & $-2.2598(0.0238)$ & $-3.2398(0.0012)$ & $-2.8698(0.0041)$ \\
CTG & $-1.8642(0.0623)$ & $-1.9876(0.0469)$ & $-1.7358(0.0826)$ \\
FDX & $-2.0123(0.0442)$ & $-2.6834(0.0073)$ & $-3.1223(0.0018)$ \\
HON & $-1.8906(0.0587)$ & $-2.3123(0.0208)$ & $-3.2590(0.0011)$ \\
HPQ & $-1.4229(0.1548)$ & $-3.5429(0.0004)$ & $-2.5960(0.0094)$ \\
IBM & $-2.6321(0.0085)$ & $-4.2343(0.0000)$ & $-5.7195(0.0000)$ \\
JPM & $-1.9990(0.0456)$ & $-2.0265(0.0427)$ & $-2.3821(0.0172)$ \\
MDZ & $-2.8359(0.0046)$ & $-4.2537(0.0000)$ & $-4.6886(0.0000)$ \\
PEP & $-2.4810(0.0131)$ & $-4.7986(0.0000)$ & $-4.1165(0.0000)$ \\
PRG & $-3.3287(0.0009)$ & $-5.0239(0.0000)$ & $-5.9189(0.0000)$ \\
TWX & $-1.6401(0.1010)$ & $-2.7771(0.0055)$ & $-2.0985(0.0359)$ \\
TXN & $-1.4331(0.1518)$ & $-3.0835(0.0020)$ & $-2.0368(0.0417)$ \\
WFC & $-1.6855(0.0919)$ & $-1.7348(0.0828)$ & $-2.3336(0.0196)$ \\
\hline
\end{tabular}

The table reports, for each stock (the ticker is given in the first column), the values of the likelihood ratio test (the $p$-values are given in brackets) proposed by Diebold and Mariano [34], at $\tau=\{0.1,0.5,0.9\}$. 
The sign of the test statistic (15) is always negative, suggesting that our approach implies a lower loss. Moreover, the performances are in most cases statistically different, given that the null hypothesis is often rejected at the $5 \%$ level. Therefore, the three tests provide clear evidence of the better performance of our method with respect to the benchmark and also in the case of single-asset realized volatilities.

To conclude, we found similar results for Models (5) and (6). In particular, for both models, the lagged value of the response variable and the lagged value of the S\&P 500 return were fundamental explanatory variables at high $\tau$ levels, which are the most critical. In contrast, the lagged value of the jump component is significant in a few cases. We determined that the relationships between four explanatory variables $\left(r r v_{i, t-1}, v i x_{t-1}, s p 500_{t-1}\right.$ and jump $\left.p_{i, t-1}\right)$ and the conditional volatility quantiles are almost always stronger in Model (5) than in Model (6). However, in the case of $\overline{r r v}_{5 i, t-1}$, the relationships are stronger in Model (6) than in Model (5). Finally, even in the single-asset analysis, the goodness of the predicted power of our approach is validated using the three tests.

\section{Conclusions}

We proposed a semi-parametric approach to model and forecast the conditional distribution of asset returns' volatility. We used the quantile regression approach, considering as predictors, variables built from the lagged values of the estimated volatility, following the HAR structure Corsi [9] developed, and macro-level variables, such as the VIX and the S\&P 500 index.

We estimated volatility using the realized range-based bias corrected bipower variation introduced by Christensen et al. [12], which is a consistent estimator of the integrated variance in the presence of microstructure noise and jumps in the context of high-frequency data. Our analyses considered 16 companies that operate in a variety of sectors in the U.S. market, and the results provide evidence of relevant impacts by the explanatory variables. In particular, the lagged values of the estimated volatility and the S\&P 500 return were critical indicators in the context of extreme events, where volatility can reach considerably high levels. These two regressors were highly significant in terms of their ability to explain the high quantiles of volatility. Moreover, the test Koenker and Bassett [54] introduced allowed us to reject the location-shift hypothesis, highlighting the heterogeneous impacts of the regressors across quantiles.

To assess the evolution of the relationships among the variables over time, we carried out a rolling analysis with steps of one day and subsamples consisting of 500 observations. Thus, we verified that two special events, the U.S. subprime crisis and the European sovereign debt crisis, have affected those relationships. In particular, acute sensitivity was recorded at high levels of quantiles. Finally, the tests developed by Berkowitz [32], Amisano and Giacomini [33] and Diebold and Mariano [34] validated the forecast performances, even in periods of financial turmoil. We compared our approach with a HARX-GJR model, which combines a HAR structure of the realized volatility mean with additional exogenous variables, and a GJR-GARCH model [30] for the mean innovation variances. The results give evidence of the superior performance of our approach.

Our findings provide supporting evidence for the use of quantile regression methods for the quantile forecasts and for the density forecast of the realized range volatility. The improvement over traditional methods is marked and will be relevant in all areas where volatility quantile values and volatility density forecasts play a role. The use of predicted volatility levels is central, for instance, in the pricing of equity derivatives, in the development of equity derivative trading strategies and in risk measurement when risk is associated with volatility. The volatility distribution is of interest when trading/pricing volatility derivatives, when designing volatility hedges for generic portfolios and when accounting for the uncertainty of volatility point forecasts.

In the present paper, we worked with multiple assets' volatilities inside a univariate framework. We left to future works the generalization of our approach to a multivariate version and its integration in returns-based volatility models where realized volatility measures represent further conditioning information sources. 
Acknowledgments: The authors thank the Editor, two anonymous reviewers, Fabio Busetti, Sandra Paterlini and all the participants of the "International Conference on Computational and Financial Econometrics", the "CIdE Workshop for PhD students in Econometrics and Empirical Economics", the seminar at the European Business School of Wiesbaden (Germany), for the helpful comments and stimulating discussions. Massimiliano Caporin acknowledges financial support from the MIUR (Ministero dell'Istruzione, dell'Universitá e della Ricerca) PRIN (Progetti di Rilevante Interesse Nazionale) project MISURA-Multivariate Statistical Models for Risk Assessment. Both authors acknowledge financial support from the University of Padova. Usual disclaimers apply.

Author Contributions: Both authors contributed equally to the paper.

Conflicts of Interest: The authors declare no conflict of interest.

\section{References}

1. Black, F.; Scholes, M. The pricing of options and corporate liabilities. J. Political Econ. 1973, 81, 637-654.

2. Engle, R.F. Autoregressive conditional heteroscedasticity with estimates of the variance of United Kingdom inflations. Econometrica 1982, 50, 987-1007.

3. Bollerslev, T. Generalized autoregressive conditional heteroskedasticity. J. Econom. 1986, 31, 307-327.

4. Melino, A.; Turnbull, S.M. Pricing foreign currency options with stochastic volatility. J. Econom. 1990, 45, 239-265.

5. Taylor, S.J. Modeling stochastic volatility: a review and comparative study. Math. Finance 1994, 4, $183-204$.

6. Harvey, A.C.; Ruiz, E.; Shephard, N. Multivariate stochastic variance models. Rev. Econ. Stud. 1994, 61, 247-264.

7. Jacquier, E.; Polson, N.; Rossi, P. Bayesian analysis of stochastic volatility models (with discussion). J. Bus. Econ. Stat. 1994, 12, 371-417.

8. Cont, R. Empirical properties of asset returns: stylized facts and statistical issues. Quant. Finance 2001, 1, 223-236.

9. Corsi, F. A simple approximate long-memory model of realized volatility. J. Financ. Econom. 2009, 7, $174-196$.

10. Andersen, T.; Bollerslev, T.; Diebold, F.X.; Labys, P. Modeling and Forecasting Realized Volatility. Econometrica 2003, 71, 579-625.

11. Martens, M.; van Dijk, D. Measuring volatility with the realized range. J. Econom. 2007, 138, 181-207.

12. Christensen, K.; Podolskij, M.; Vetter, M. Bias-correcting the realized range-based variance in the presence of market microstructure noise. Finance Stoch. 2009, 13, 239-268.

13. Hasbrouck, J. Empirical Market Microstructure. The Institutions, Economics, and Econometrics of Securities Trading; Oxford University Press: New York, NY, USA, 2006.

14. O'Hara, M. Market Microstructure Theory; Blackwell: Cambridge, UK, 1998.

15. Roll, R. A simple model of the implicit bid-ask spread in an efficient market. J. Finance 1984, 39, 1127-1139.

16. Christiansen, C.; Schmeling, M.; Schrimpf, A. A comprehensive look at financial volatility prediction by economic variables. J. Appl. Econom. 2012, 27, 956-977.

17. Paye, B.S. Déjà vol: Predictive regressions for aggregate stock market volatility using macroeconomic variables. J. Financ. Econ. 2012, 106, 527-546.

18. Fernandes, M.; Medeiros, M.C.; Scharth, M. Modeling and Predicting the Cboe Market Volatility Index; Textos para discussão, Escola de Economia de São Paulo, Getulio Vargas Foundation (Brazil) No. 342, 2009.

19. Caporin, M.; Velo, G. Modeling and Forecasting Realized Range Volatility; Marco Fanno Working Paper No. 128-2011; SSRN: Rochester, NY, USA, 2011.

20. Caporin, M.; Rossi, E.; de Magistris, P.S. Conditional Jumps in Volatility and Their Economic Determinants; SSRN: Rochester, NY, USA, 2011.

21. Opschoor, A.; Dijk, D.V.; der Wel, M.V. Predicting volatility and correlations with Financial Conditions Indexes. J. Empir. Finance 2014, 29, 435-447.

22. Koenker, R.; Bassett, G. Regression quantiles. Econometrica 1978, 46, 33-50.

23. Engle, F.; Manganelli, S. CAViaR: Conditional Autoregressive Value at Risk by Regression Quantiles. J. Bus. Econ. Stat. 2004, 22, 367-381.

24. White, H.; Kim, T.H.; Manganelli, S. Modeling Autoregressive Conditional Skewness and Kurtosis with Multi-Quantile CAViaR; Working Paper Series 0957; European Central Bank: Frankfurt am Main, Germany, 2008. 
25. White, H.; Kim, T.H.; Manganelli, S. VAR for VaR: Measuring Systemic Risk Using Multivariate Regression Quantiles; MPRA Paper 35372; University Library of Munich: Munich, Germany, 2010.

26. Li, M.L.; Miu, P. A hybrid bankruptcy prediction model with dynamic loadings on accounting-ratio-based and market-based information: A binary quantile regression approach. J. Empir. Finance 2010, 17, 818-833.

27. Castro, C.; Ferrari, S. Measuring and testing for the systemically important financial institutions. J. Empir. Finance 2014, 25, 1-14.

28. Caporin, M.; Pelizzon, L.; Ravazzolo, F.; Rigobon, R. Measuring Sovereign Contagion in Europe; NEBR Working paper No. 18741; National Bureau of Economic Research: Cambridge, MA, USA, 2014.

29. Zikes, F.; Barunik, J. Semiparametric Conditional Quantile Models for Financial Returns and Realized Volatility. J. Financial Econom. 2016, 14, 185-226.

30. Glosten, L.R.; Jagannathan, R.; Runkle, D.E. On the relation between the expected value and the volatility of nominal excess return on stocks. J. Finance 1993, 48, 1779-1801.

31. Corsi, F.; Mittnik, S.; Pigorsch, C.; Pigorsch, U. The volatility of realized volatility. Econom. Rev. 2008, $27,46-78$.

32. Berkowitz, J. Testing density forecasts, with applications to risk management. J. Bus. Econ. Stat. 2001, 19, 465-474.

33. Amisano, G.; Giacomini, R. Comparing Density Forecasts via Weighted Likelihood Ratio Tests. J. Bus. Econ. Stat. 2007, 25, 177-190.

34. Diebold, F.; Mariano, R. Comparing Predictive Accuracy. J. Bus. Econ. Stat. 2002, 20, 134-144.

35. Zhang, J.E.; Shu, J.; Brenner, M. The new market for volatility trading. J. Futures Markets 2010, 30, 809-833.

36. Euan, S. Volatility Trading, 2nd ed.; John Wiley \& Sons Inc.: Hoboken, NJ, USA, 2013.

37. Shephard, N.; Sheppard, K.K. Realising the future: Forecasting with high-frequency-based volatility (HEAVY) models. J. Appl. Econom. 2010, 25, 197-231.

38. Nelson, C.R.; Plosser, I.C. Trends and random walks in macroeconomic time series : Some evidence and implications. J. Monet. Econ. 1982, 10, 139-162.

39. Dickey, D.; Fuller, W.A. Likelihood Ratio Statistics for Autoregressive Time Series with a Unit Root. Econometrica 1981, 49, 1057-1072.

40. Phillips, P.C.B.; Perron, P. Testing for a Unit Root in Time Series Regression. Biometrika 1988, 75, 335-346.

41. Christensen, K.; Podolskij, M. Range-Based Estimation of Quadratic Variation; Technical Reports 2006,37; Technische Universität Dortmund, Sonderforschungsbereich 475: Dortmund, Germany, 2006.

42. Christensen, K.; Podolskij, M. Realized range-based estimation of integrated variance. J. Econom. 2007, 141, 323-349.

43. Koenker, R. A note on L-estimators for linear models. Stat. Probab. Lett. 1984, 2, 323-325.

44. Bondell, H.; Reich, B.; Wang, H. Non-crossing quantile regression curve estimation. Biometrika 2010, 97, 825-838.

45. Zhao, Y. Real-Time Density Forecasts of Output and Inflation via Quantile Regression; Working Paper Series 1302; East Carolina University, 2013.

46. Chernozhukov, V.; Fernandez-Val, I.; Galichon, A. Quantile and probability curves without crossing. Econometrica 2010, 78, 1093-1125.

47. Gaglianone, W.; Lima, L. Constructing Density Forecasts from Quantile Regressions. J. Money Credit Bank. 2012, 44, 1589-1607.

48. Rosenblatt, M. Remarks on a multivariate transformation. Ann. Math. Stat. 1952, 23, 470-472.

49. Newey, W.K.; West, K.D. A Simple, Positive Semidefinite, Heteroskedasticity and Autocorrelation Consistent Covariance Matrix. Econometrica 1987, 55, 703-708.

50. Koenker, R.; Machado, J.A.F. Goodness of fit and related inference process for quantile regression. J. Am. Stat. Assoc. 1999, 94, 1296-1310.

51. Koenker, R.; Bassett, G. Tests of linear hypotheses and $l_{1}$ estimation. Econometrica 1982, 50, 1577-1583.

52. Corsi, F.; Pirino, D.; Reno, R. Threshold bipower variation and the impact of jumps on volatility forecasting. J. Econom. 2010, 159, 276-288. 
53. Black, F. Studies of Stock Market Volatility Changes. In Proceedings of the 1976 Meeting of the Business and Economic Statistics Section; American Statistical Association: Washington, DC, USA, 1976; pp. 177-181.

54. Koenker, R.; Bassett, G. Robust tests for heteroscedasticity based on regression quantiles. Econometrica 1982, $50,43-61$.

(C) 2016 by the authors; licensee MDPI, Basel, Switzerland. This article is an open access article distributed under the terms and conditions of the Creative Commons Attribution (CC-BY) license (http://creativecommons.org/licenses/by/4.0/). 